\title{
Explicit iteration and unbounded solutions for fractional $q$-difference equations with boundary conditions on an infinite interval
}

\author{
Abdellatif Boutiara', Maamar Benbachir², Mohammed K.A. Kaabar ${ }^{3 *}$, Francisco Martínez ${ }^{4}$, Mohammad \\ Esmael Samei ${ }^{* *}$ iD and Melike Kaplan ${ }^{6}$
}

\author{
${ }^{*}$ Correspondence: \\ mohammed.kaabar@wsu.edu: \\ mesamei@gmail.com; \\ mesamei@basu.ac.ir \\ ${ }^{3}$ Institute of Mathematical Sciences, \\ Faculty of Science, University of \\ Malaya, Kuala Lumpur, 50603, \\ Malaysia \\ ${ }^{5}$ Department of Mathematics, Bu-Ali \\ Sina University, Hamedan, Iran \\ Full list of author information is \\ available at the end of the article
}

\begin{abstract}
In this work, a proposed system of fractional boundary value problems is investigated concerning its unbounded solutions' existence for a class of nonlinear fractional q-difference equations in the context of the Riemann-Liouville fractional q-derivative on an infinite interval. The system's solution is formulated with the help of Green's function. A compactness criterion is established in a special space. All the obtained results of uniqueness and existence are investigated with the help of fixed-point theorems. Some essential examples are illustrated to support our main outcomes.
\end{abstract}

MSC: 26A33; 34A08; 34B25

Keywords: Fractional q-difference equation; Fractional q-derivative; Unbounded solution; Explicit iteration; Infinite interval

\section{Introduction}

Fractional differential equations are much better than integer ones with respect to their nature in the descriptions of phenomena and processes of several scientific and engineering phenomena. Various studies of fractional differential equations have been recently conducted in the context of fractional-order derivatives such as the Riemann-Liouville fractional derivative and the Caputo fractional derivative.

Fractional calculus and q-calculus in general, and fractional differential equations in particular, are well established in current research works. Fractional calculus belongs to a large portion of mathematical analysis. The notion of q-difference equations goes back to 1910, where it was introduced by Jackson [1]. In the past decades, the subject has attracted many authors, and q-difference equations have appeared as a promising research field, on both applied and theoretical levels (see [2-7]). For more details on this subject, refer to [8-20].

Recently, fractional boundary value problems on both bounded and unbounded domains have been extensively investigated (see [21-29]). On the one hand, a large number of research works on fractional calculus are dedicated to the fractional differential equations' solvability on a finite interval. On the other hand, fractional boundary value

(c) The Author(s) 2022. This article is licensed under a Creative Commons Attribution 4.0 International License, which permits use sharing, adaptation, distribution and reproduction in any medium or format, as long as you give appropriate credit to the original author(s) and the source, provide a link to the Creative Commons licence, and indicate if changes were made. The images or other third party material in this article are included in the article's Creative Commons licence, unless indicated otherwise in a credit line to the material. If material is not included in the article's Creative Commons licence and your intended use is not permitted by statutory regulation or exceeds the permitted use, you will need to obtain permission directly from the copyright holder. To view a copy of this licence, visit http://creativecommons.org/licenses/by/4.0/. 
problems on an infinite interval have been rarely studied (see [30-38]). Arara et al. [30] investigated the bounded solutions' existence for fractional differential equations in the sense of the Caputo fractional derivative on the unbounded domain expressed as:

$$
\left\{\begin{array}{l}
{ }^{c} \mathbb{D}_{0}^{S} \wp(\mathfrak{t})=\mathfrak{w}(\mathfrak{t}, \wp(\mathfrak{t})), \quad \mathfrak{t} \geq 0, \\
\wp(0)=\wp_{0}, \\
\wp \text { is bounded on } J:=[0, \infty),
\end{array}\right.
$$

where $\varsigma \in(1,2),{ }^{c} \mathbb{D}_{0^{+}}^{\varsigma}$ is the Caputo fractional derivative of order $\varsigma, \wp_{0} \in \mathbb{R}$, and $\mathfrak{w}$ : $J \times \mathbb{R} \rightarrow \mathbb{R}$ is continuous. From a combined Schauder fixed-point theorem with a diagonalization method, $\mathrm{Su}$ [31] discussed the solutions' existence for the following fractional boundary value problem:

$$
\left\{\begin{array}{l}
\mathbb{D}_{0^{+}}^{S} \wp(\mathfrak{t})=\mathfrak{w}(\mathfrak{t}, \wp(\mathfrak{t})), \quad \mathfrak{t} \in J, \\
\wp(0)=0, \quad \mathbb{D}_{0^{+}}^{\varsigma-1} \wp(\infty)=\wp_{\infty},
\end{array}\right.
$$

where $1<\varsigma \leq 2, \mathfrak{w} \in \mathcal{C}(j \times \mathbb{E}, \mathbb{E}), \wp_{\infty} \in \mathbb{E}, \mathbb{D}_{0^{+}}^{\varsigma}$ and $\mathbb{D}_{0^{+}}^{\zeta-1}$ are Riemann-Liouville fractional derivatives. The applied technique relies on the Kuratowski measure properties of noncompactness and the Darbo fixed-point theorem on infinite intervals in Banach space. Zhao et al. [32] proved the positive solutions' existence for the following fractional differential equation:

$$
\left\{\begin{array}{l}
\mathbb{D}_{0+}^{\varsigma} \wp(\mathfrak{t})+\mathfrak{w}(\mathfrak{t}, \wp(\mathfrak{t}))=0, \quad \mathfrak{t} \in J, \\
\wp(0)=0, \quad \lim _{\mathfrak{t} \rightarrow+\infty} \mathbb{D}_{0+}^{\varsigma-1} \wp(\mathfrak{t})=\beta \wp(v),
\end{array}\right.
$$

via the Leray-Schauder nonlinear alternative theorem, where $1<\varsigma \leq 2, \mathfrak{w} \in \mathcal{C}(J \times$ $\mathbb{R}, \jmath), 0 \leq v<\infty$ and $\mathbb{D}_{0+}^{5}$ is the usual Riemann-Liouville fractional derivative. Zhao et al. [2] investigated the fractional multipoint boundary value problem:

$$
\left\{\begin{array}{l}
\mathbb{D}_{0^{+}}^{\varsigma} \wp(\mathfrak{t})+\mathfrak{w}(\mathfrak{t}, \wp(\mathfrak{t}))=0, \quad \mathfrak{t} \in J \\
\wp(0)=0, \quad \mathbb{D}_{0^{+}}^{S-1} \wp(+\infty)=\sum_{i=1}^{m} \varsigma_{i} \wp\left(v_{i}\right),
\end{array}\right.
$$

where $1<\varsigma \leq 2,0<v_{1}<v_{2}<\cdots<v_{m}<\infty, \mathbb{D}_{0^{+}}^{\varsigma}$ is a usual Riemann-Liouville fractional derivative. Three positive solutions' existence was proven via the Leggett-Williams fixedpoint theorem. Rezapour et al. investigated the multisingular fractional integrodifferential pointwise equation

$$
\left\{\begin{array}{l}
\mathbb{D}_{\mathrm{q}}^{5} \wp(\mathfrak{t})=\mathfrak{w}\left(\mathfrak{t}, \wp(\mathfrak{t}), \wp^{\prime}(\mathfrak{t}), \mathbb{D}^{\gamma_{1}} \wp(\mathfrak{t}), \mathbb{I}^{\gamma_{1}} \wp(\mathfrak{t})\right), \quad(\forall \mathfrak{t} \in[0,1]), \\
\wp^{\prime}(0)=\wp(a), \quad \wp(1)=\int_{0}^{b} \wp(\xi) \mathrm{d} \xi
\end{array}\right.
$$

$\wp^{(j)}(0)=0 j=2, \ldots,[\sigma]-1$, where $\wp \in C^{1}([0,1]), \gamma_{1}, \gamma_{2} \in[2, \infty), a, b \in(0,1), \mathbb{D}^{\varsigma}$ is the Caputo fractional q-derivative of ord $\zeta$, and $\mathfrak{w}:[0,1] \times \mathbb{R}^{4} \rightarrow \mathbb{R}$ is a function such that $\mathfrak{w}(\mathfrak{t}, \cdot, \cdot, \cdot, \cdot)$ is singular at some points $\mathfrak{t} \in[0,1][6]$. The authors in [24] applied the LeraySchauder nonlinear alternative theorem and Leggett-Williams fixed-point theorem to 
prove the positive solutions' existence to the following fractional differential equation:

$$
\left\{\begin{array}{l}
\left(\mathbb{D}_{\mathrm{q}}^{S} \wp\right)(\mathfrak{t})+\mathfrak{w}(\mathfrak{t}, \wp(\mathfrak{t}))=0, \quad t \in J \\
\wp(0)=0, \quad \mathbb{D}_{\mathrm{q}}^{S-1} \wp(+\infty)=\sum_{i=1}^{m} a_{i} \wp\left(v_{i}\right),
\end{array}\right.
$$

where $0<\mathrm{q}<1,1<5<2$,

$$
0 \leq \sum_{i=1}^{m} a_{i} v_{i}^{\varsigma-1}<\Gamma_{\mathrm{q}}(\varsigma)
$$

$\mathbb{D}_{\mathrm{q}}^{S}$ is a Riemann-Liouville fractional q-derivative. $\mathfrak{w}:(j, \mathbb{R}) \rightarrow J$ is a continuous function. Zhao et al. [32] applied the Altman fixed-point theorem to study the solutions' existence to the following fractional differential equation:

$$
\left\{\begin{array}{l}
\mathbb{D}^{\varsigma} \wp(\mathfrak{t})+\mathfrak{w}(\mathfrak{t}, \wp(\mathfrak{t}), \mathcal{T} \wp(\mathfrak{t}))=0, \quad 3<\varsigma \leq 4, \\
\wp(0)=\wp^{\prime}(0)=\wp^{\prime \prime}(0)=0, \\
\mathbb{D}^{\varsigma-1} \wp(\infty)=v \mathbb{I}^{\zeta} \wp(\eta), \quad \zeta>0,
\end{array}\right.
$$

where $\mathfrak{t} \in J, \mathfrak{w} \in \mathcal{C}\left[J \times \mathbb{R}^{2}, \mathbb{R}\right], v \in \mathbb{R}, \eta \in J, \mathbb{D}^{\varsigma}$ is the Riemann-Liouville fractional derivative of order $\zeta$, $\mathbb{I}^{\zeta}$ is the Riemann-Liouville fractional integral of order $\zeta$ and

$$
\mathcal{T} \wp(\mathfrak{t})=\int_{0}^{\mathfrak{t}} \mathbb{G}(\mathfrak{t}, \xi) \wp(\xi) \mathrm{d} \xi,
$$

with $\mathbb{G}(\mathfrak{t}, \mathfrak{s}) \in \mathcal{C}[\mathcal{E}, \mathbb{R}], \mathcal{E}=\left\{(\mathfrak{t}, \mathfrak{s}) \in \mathbb{R}^{2}: 0 \leq \mathfrak{s} \leq \mathfrak{t}\right\}$. In [38], Matar et al. considered the FDE

$$
\left\{\begin{array}{l}
\frac{\mathrm{d}}{\mathrm{d} \mathfrak{t}}\left(\varphi_{p}\left({ }^{C} \mathbb{D}^{\varsigma 1, \rho} \wp(\mathfrak{t})\right)\right)=\mathfrak{w}\left(\mathfrak{t}, \wp(\mathfrak{t}),{ }^{C} \mathbb{D}^{\varsigma 2, \rho} \wp(\mathfrak{t})\right), \quad(\forall \mathfrak{t} \in[0,1]), \\
\wp(0)+\mu \wp(1)=\mathfrak{v}_{1}(\wp(0), \wp(1)), \quad(\mu \neq 1), \\
\wp^{\prime}(1)=\mathfrak{v}_{2}(\wp(0), \wp(1)),
\end{array}\right.
$$

here ${ }^{C} \mathbb{D}^{\zeta_{1}, \rho}$ and ${ }^{C} \mathbb{D}^{S_{2}, \rho} \wp(\mathfrak{t})$ are the generalized Caputo fractional derivative of order $1<$ $\sigma_{1}<2$ and $0<\sigma_{2}<1$, respectively, $\rho>1, \varphi_{p}(p>1)$ is a $p$-Laplacian operator and the nonlinear functions $\mathfrak{w}:[0,1] \times \mathbb{R}^{2} \rightarrow \mathbb{R}$ and $\mathfrak{v}_{1}, \mathfrak{v}_{2}: \mathbb{R}^{2} \rightarrow \mathbb{R}$ are given continuous functions.

The solutions' existence for the following boundary value problem of nonlinear fractional q-difference equations on an infinite interval are investigated in this work:

$$
\left\{\begin{array}{l}
\mathbb{D}_{\mathrm{q}}^{\zeta} \wp(\mathfrak{t})+\mathfrak{w}(\mathfrak{t}, \wp(\mathfrak{t}))=0, \quad 3<\varsigma \leq 4, \\
\wp(0)=\wp^{\prime}(0)=\wp^{\prime \prime}(0)=0, \\
\mathbb{D}_{\mathrm{q}}^{\zeta-1} \wp(\infty)=\sum_{i=1}^{m} \mathrm{a}_{i} \wp\left({ }_{1} \eta_{i}\right)+\sum_{i=1}^{m} \mathrm{a}_{i} \mathbb{D}_{\mathrm{q}}^{v} \wp\left({ }_{2} \eta_{i}\right), \quad v>0,
\end{array}\right.
$$

where $\mathfrak{t} \in J, \mathfrak{w} \in \mathcal{C}(j \times \mathbb{R}, \mathbb{R}), 0<{ }_{1} \eta_{i},{ }_{2} \eta_{i}<+\infty, i=1, \ldots, m, \mathbb{D}_{\mathrm{q}}^{(.)}$is the Riemann-Liouville fractional q-derivative with $0<\mathrm{q}<1,{ }_{1} \mathrm{a}_{i},{ }_{2} \mathrm{a}_{i}>0$ such that

$$
\left\{\sum_{i=1}^{m}{ }_{1} \mathrm{a}_{i 1} \eta_{i}^{\varsigma-1}+\frac{\Gamma_{\mathrm{q}}(\varsigma)}{\Gamma_{\mathrm{q}}(\varsigma-v)} \sum_{i=1}^{m}{ }_{2} \mathrm{a}_{i 2} \eta_{i}^{\varsigma-\nu-1}\right\} \leq \Gamma_{\mathrm{q}}(\varsigma),
$$

and $\mathfrak{w}:(\jmath, \mathbb{R}) \rightarrow \mathbb{R}$ is a continuous function. 
A variable's range is studied in this research work on the unbounded domain. A modified compactness criterion is utilized to investigate the studied compact operator $\mathfrak{H}$ due to the failure of the Arzelá-Ascoli theorem in space $\mathcal{C}_{\infty}$. With the help of the Banach fixed-point theorem, the Leray-Schauder nonlinear alternative theorem, and the Schauder fixedpoint theorem, the investigated system's uniqueness and existence are proven.

The remainder of this article is organized as follows: the main definitions and lemmas are discussed in Sect. 2 to offer a guide for proving our results by providing a necessary background with some properties to formulate a Green's function that is suitable for the investigated problem on an unbounded domain. In Sect. 3, the solution's existence and uniqueness are investigated for the boundary value problem (4). In Sect. 4, two examples are presented to apply our outcomes.

\section{Preliminaries}

Essential q-derivative and q-integral notions are presented in this section. For more background information, refer to $[4,11,12,33,39,40]$. Consider the real number $\mathrm{v}$ and put $[\mathrm{v}]_{\mathrm{q}}=\left(1-\mathrm{q}^{\mathrm{v}}\right)(1-\mathrm{q})^{-1}$. The $\mathrm{q}$-analog of the power $(\mathrm{v}-\hat{\mathrm{v}})^{l}$ is $(\mathrm{v}-\hat{\mathrm{v}})^{(0)}=1$,

$$
(\mathrm{v}-\hat{\mathrm{v}})^{(l)}=\prod_{\kappa=0}^{l-1}\left(\mathrm{v}-\mathrm{vq}^{\kappa}\right), \quad(\mathrm{v}, \mathrm{v} \in \mathbb{R}, l \in \mathbb{N}) .
$$

Generally, $\forall \mathrm{v}$, v́ and $\varsigma \in \mathbb{R}$,

$$
(\mathrm{v}-\hat{\mathrm{v}})^{(\varsigma)}=\mathrm{v}^{\varsigma} \prod_{\kappa=0}^{\infty}\left(\frac{\mathrm{v}-\mathbf{v}^{\kappa}}{\mathrm{v}-\mathbf{v}^{\kappa+\zeta}}\right) .
$$

The q-gamma function is expressed as $\Gamma_{\mathrm{q}}(\varsigma)=(1-\mathrm{q})^{(\varsigma-1)}(1-\mathrm{q})^{1-\varsigma}$, for $\varsigma \in \mathbb{R}-$ $\{\ldots,-2,-1,0\}$ [39]. The q-gamma function satisfies $\left(\Gamma_{\mathrm{q}}(1+\varsigma)=[\varsigma]_{\mathrm{q}} \Gamma_{\mathrm{q}}(\varsigma)\right)$. For any $\varsigma, v>0$, the q-beta function is expressed as

$$
\mathbb{B}_{\mathrm{q}}(\varsigma, \nu)=\int_{0}^{1} \xi^{(\varsigma-1)}(1-\mathrm{q} \xi)^{(\nu-1)} \mathrm{d}_{\mathrm{q}} \xi, \quad \mathrm{q} \in(0,1),
$$

where the q-beta function's expression in terms of the q-gamma function is ([39])

$$
\mathbb{B}_{\mathrm{q}}(\varsigma, v)=\frac{\Gamma_{\mathrm{q}}(\varsigma) \Gamma_{\mathrm{q}}(v)}{\Gamma_{\mathrm{q}}(\varsigma+v)} .
$$

The q-derivative of order $\imath \in \mathbb{N}$, of a function $\wp$ is expressed as $\mathbb{D}_{\mathrm{q}}^{0} \wp(\mathfrak{t})=\wp(\mathfrak{t})$,

$$
\begin{aligned}
\mathbb{D}_{\mathrm{q}} \wp(\mathfrak{t}):=\mathbb{D}_{\mathrm{q}}^{1} \wp(\mathfrak{t})=\frac{\wp(\mathfrak{t})-\wp(\mathrm{q} \mathfrak{t})}{(1-\mathrm{q}) \mathfrak{t}}, \quad(\mathfrak{t} \neq 0), \\
\mathbb{D}_{\mathrm{q}} \wp(0)=\lim _{\mathfrak{t} \rightarrow 0} \mathbb{D}_{\mathrm{q}} \wp(\mathfrak{t}), \text { and } \mathbb{D}_{\mathrm{q}}^{\iota} \wp(\mathfrak{t})=\mathbb{D}_{\mathrm{q}} \mathbb{D}_{\mathrm{q}}^{l-1} \wp(\mathfrak{t}), \forall \mathfrak{t} \in J \text { [39]. Set } \\
J_{\mathfrak{t}}:=\left\{\mathfrak{t q}^{l}: \iota \in \mathbb{N}\right\} \cup\{0\} .
\end{aligned}
$$

The q-integral of a function $\wp: \jmath_{\mathfrak{t}} \rightarrow \mathbb{R}$ is written as

$$
\mathbb{I}_{\mathrm{q}} \wp(\mathfrak{t})=\int_{0}^{\mathfrak{t}} \wp(\xi) \mathrm{d}_{\mathrm{q}} \xi=\sum_{\kappa=0}^{\infty} \mathfrak{t}(1-\mathrm{q}) \mathrm{q}^{\kappa} \wp\left(\mathfrak{t q}^{\kappa}\right),
$$


iff the series converges [39]. Obviously, $\mathbb{D}_{\mathbf{q}} \mathbb{I}_{\mathbf{q}} \wp(\mathfrak{t})=\wp(\mathfrak{t})$. Suppose that $\wp$ is continuous at 0 , then $\mathbb{I}_{\mathrm{q}} \mathbb{D}_{\mathrm{q}} \wp(\mathfrak{t})=\wp(\mathfrak{t})-\wp(0)$ [39]. The fractional q-integral of the Riemann-Liouville type of order $\alpha \geq 0$, of a function $\wp: \mathbb{I} \rightarrow \mathbb{R}$ is expressed as $\mathbb{I}_{\mathfrak{q}}^{0} \wp(\mathfrak{t})=\wp(\mathfrak{t})$, and $\forall \mathfrak{t} \in \mathbb{I}$, [9]

$$
\mathbb{I}_{\mathrm{q}}^{\varsigma} \wp(\mathfrak{t})=\int_{0}^{\mathfrak{t}} \frac{(\mathfrak{t}-\mathrm{q} \xi)^{(\varsigma-1)}}{\Gamma_{\mathrm{q}}(\varsigma)} \wp(\xi) \mathrm{d}_{\mathrm{q}} \xi
$$

Lemma 2.1 ([4]) Assume that $\varsigma, v \geq 0$, and $\wp$ is a function defined on $[0,1]$. Then, the following hold $\mathbb{I}_{\mathrm{q}}^{S} \mathbb{I}_{\mathrm{q}}^{v} \wp(\mathfrak{t})=\mathbb{I}_{\mathrm{q}}^{S+v} \wp(\mathfrak{t})=\mathbb{I}_{\mathrm{q}}^{v} \mathbb{I}_{\mathrm{q}}^{\zeta} \wp(\mathfrak{t})$, and

$$
\mathbb{I}_{\mathrm{q}}^{\varsigma} \mathfrak{t}^{v}=\frac{\Gamma_{\mathrm{q}}(v+1)}{\Gamma_{\mathrm{q}}(\varsigma+v+1)} \mathfrak{t}^{\varsigma+v}, \quad \forall v \in(-1, \infty), \varsigma \in J, \mathfrak{t}>0 .
$$

In particular, if $\wp \equiv 1$, then $\mathbb{I}_{\mathrm{q}}^{\zeta} 1(\mathfrak{t})=\frac{1}{\Gamma_{\mathrm{q}}(1+\varsigma)} \mathfrak{t}^{(\varsigma)}, \forall \mathfrak{t}>0$.

Definition 2.2 ([40]) The fractional q-derivative of the Riemann-Liouville type of order $\varsigma \geq 0$, of a function $\wp: \jmath \rightarrow \mathbb{R}$ is defined by $\mathbb{D}_{\mathrm{q}}^{0} \wp(\mathfrak{t})=\wp(\mathfrak{t})$, and

$$
\mathbb{D}_{\mathrm{q}}^{\zeta} \wp(\mathfrak{t})=\mathbb{D}_{\mathrm{q}}^{[\varsigma]} \mathbb{I}_{\mathrm{q}}^{[\zeta]-\varsigma} \wp(\mathfrak{t})=\frac{1}{\Gamma_{\mathrm{q}}(l-\varsigma)} \int_{0}^{\mathfrak{t}} \frac{\wp((\xi)}{(\mathfrak{t}-\mathrm{q} \xi)^{\varsigma-l+1}} \mathrm{~d}_{\mathrm{q}} \xi
$$

where $l=[\varsigma]$ is the integer part of $\varsigma$.

Lemma 2.3 ([12]) Assume that $\varsigma>0$ and $\iota \in \mathbb{N}$. Then, an equality holds as follows:

$$
\mathbb{I}_{\mathrm{q}}^{\varsigma} \mathbb{D}_{\mathrm{q}}^{l} \wp(\mathfrak{t})=\mathbb{D}_{\mathrm{q}}^{l} \mathbb{I}_{\mathrm{q}}^{\varsigma} \wp(\mathfrak{t})-\sum_{\kappa=0}^{\zeta-1} \frac{\mathfrak{t}^{\zeta-l+\kappa}}{\Gamma_{\mathrm{q}}(\varsigma+\kappa-\imath+1)}\left(\mathbb{D}_{\mathrm{q}}^{\kappa} \wp\right)(0)
$$

Obviously, we have:

$$
\mathbb{D}_{\mathrm{q}}^{\zeta} \mathfrak{t}^{\nu-1}=\frac{\Gamma_{\mathrm{q}}(v)}{\Gamma_{\mathrm{q}}(v-\varsigma)} \mathfrak{t}^{\nu-\varsigma-1}
$$

Lemma 2.4 ([11]) Assume that $J_{0}$ and $\mathrm{J}$ are intervals involving $0 \in J_{0} \subseteq J_{\mathrm{J}}$. Assume that $\wp_{l},(l \in \mathbb{N})$ and $\wp$ are functions defined in $J \lim _{l \rightarrow \infty} \wp_{l}(\mathfrak{t})=\wp(\mathfrak{t}),(\forall \mathfrak{t} \in J)$, and $\wp_{l}$ tends uniformly to $\wp$ on $J_{0}$. Then,

$$
\lim _{l \rightarrow \infty} \int_{0}^{\mathrm{v}} \wp_{l}(\xi) \mathrm{d}_{\mathrm{q}} \xi=\int_{0}^{\mathrm{v}} \wp(\xi) \mathrm{d}_{\mathrm{q}} \xi \quad(\forall \mathrm{v} \in J) .
$$

The following results are important in the remainder of the paper, where we give the exact expression of the Green's function associated with the system (4).

Lemma 2.5 A given function: $\hbar \in \mathcal{C}\left(\mathbb{R}^{+}\right)$is assumed. Then, the boundary value problem:

$$
\mathbb{D}_{\mathrm{q}}^{S} \wp(\mathfrak{t})+\hbar(\mathfrak{t})=0, \quad \mathfrak{t} \in J
$$


via boundary conditions

$$
\left\{\begin{array}{l}
\wp(0)=\wp^{\prime}(0)=\wp^{\prime \prime}(0)=0 \\
\mathbb{D}_{\mathrm{q}}^{S-1} \wp(+\infty)=\sum_{i=1}^{m} \mathrm{a}_{i} \wp\left({ }_{1} \eta_{i}\right)+\sum_{i=1}^{m} \mathrm{a}_{i} \mathbb{D}_{\mathrm{q}}^{v} \wp\left({ }_{2} \eta_{i}\right)
\end{array}\right.
$$

has a unique solution

$$
\begin{aligned}
\wp(\mathfrak{t})= & \int_{0}^{\infty}{ }_{1} \mathbb{G}_{\mathrm{q}}(\mathfrak{t}, \xi) \hbar(\xi) \mathrm{d}_{\mathrm{q}} \xi \\
& +\frac{\mathfrak{t}^{\varsigma-1}}{\Lambda} \sum_{i=1}^{m}{ }_{1} \mathrm{a}_{i} \int_{0}^{\infty}{ }_{2} \mathbb{G}_{\mathrm{q}}\left({ }_{1} \eta_{i}, \xi\right) \hbar(\xi) \mathrm{d}_{\mathrm{q}} \xi \\
& +\frac{\mathfrak{t}^{5-1}}{\Lambda} \sum_{i=1}^{m}{ }_{2} \mathrm{a}_{i} \int_{0}^{\infty}{ }_{2} \mathbb{G}_{\mathrm{q}}\left({ }_{2} \eta_{i}, \xi\right) \hbar(s) \mathrm{d}_{\mathrm{q}} \xi
\end{aligned}
$$

with $\Lambda$ defined by

$$
\Lambda=\left\{\Gamma_{\mathrm{q}}(\varsigma)-\left(\sum_{i=1}^{m}{ }_{1} \mathrm{a}_{i 1} \eta_{i}^{\varsigma-1}+\frac{\Gamma_{\mathrm{q}}(\varsigma)}{\Gamma_{\mathrm{q}}(\varsigma-v)} \sum_{i=1}^{m}{ }_{2} \mathrm{a}_{i 2} \eta_{i}^{\varsigma-\nu-1}\right)\right\}
$$

where

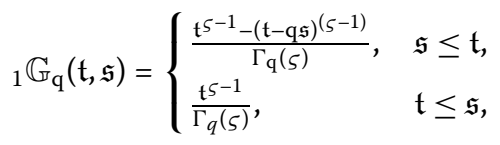

and

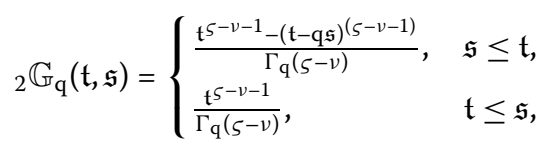

$\forall \mathfrak{t}, \mathfrak{s} \in \mathrm{J}$ is the Green's function of boundary value problem (6) and (7).

Proof By integrating both sides of (6)

$$
\mathbb{I}_{\mathrm{q}}^{\varsigma} \mathbb{D}_{\mathrm{q}}^{\varsigma} \wp(\mathfrak{t})=-\mathbb{I}_{\mathrm{q}}^{\varsigma} \hbar(\mathfrak{t})
$$

The Definition 2.2 and Lemma 2.3, imply that

$$
\wp(\mathfrak{t})=-\frac{1}{\Gamma_{\mathrm{q}}(\varsigma)} \int_{0}^{\mathfrak{t}}(\mathfrak{t}-\mathrm{q} \xi)^{(\varsigma-1)} \hbar(\xi) \mathrm{d}_{\mathrm{q}} \xi+c_{1} \mathfrak{t}^{\varsigma-1}+c_{2} \mathfrak{t}^{\varsigma-2}+c_{3} \mathfrak{t}^{\varsigma-3}+c_{4} \mathfrak{t}^{\varsigma-4}
$$

The boundary conditions implies that $\wp(0)=\wp^{\prime}(0)=\wp^{\prime \prime}(0)=0$, thus,

$$
c_{2}=c_{3}=c_{4}=0 \text {, }
$$

hence,

$$
\wp(\mathfrak{t})=-\frac{1}{\Gamma_{\mathrm{q}}(\varsigma)} \int_{0}^{\mathfrak{t}}(\mathfrak{t}-\mathrm{q} \xi)^{(\varsigma-1)} \hbar(\xi) \mathrm{d}_{\mathrm{q}} \xi+c_{1} \mathfrak{t}^{\varsigma-1}
$$


We obtain:

$$
\begin{aligned}
\sum_{i=1}^{m}{ }_{1} \mathrm{a}_{i} \wp\left({ }_{1} \eta_{i}\right)= & c_{1} \sum_{i=1}^{m}{ }_{1} \mathrm{a}_{i 1} \eta_{i}^{\varsigma-1} \\
& \left.-\frac{1}{\Gamma_{\mathrm{q}}(\varsigma)} \sum_{i=1}^{m}{ }_{1} \mathrm{a}_{i} \int_{0}{ }^{1}{ }^{1}{ }_{1}{ }_{1} \eta_{i}-\mathrm{q} \xi\right)^{(\varsigma-1)} \hbar(\xi) \mathrm{d}_{\mathrm{q}} \xi
\end{aligned}
$$

By differentiating both sides of (10) along with (5), we obtain

$$
\begin{aligned}
\sum_{i=1}^{m}{ }_{2} \mathrm{a}_{i} \mathbb{D}_{\mathrm{q}}^{v} \wp\left({ }_{2} \eta_{i}\right)= & c_{1} \sum_{i=1}^{m}{ }_{2} \mathrm{a}_{i} \frac{\Gamma(\varsigma)}{\Gamma(\varsigma-v)}{ }^{2} \eta_{i}^{\zeta-\nu-1} \\
& \left.-\frac{1}{\Gamma_{\mathrm{q}}(\varsigma-v)} \sum_{i=1}^{m}{ }_{2} \mathrm{a}_{i} \int_{0}{ }^{2 \eta_{i}}{ }_{2} \eta_{i}-\mathrm{q} \xi\right)^{(\varsigma-1)} \hbar(\xi) \mathrm{d}_{\mathrm{q}} \xi
\end{aligned}
$$

and $\mathbb{D}_{\mathrm{q}}^{\zeta-1} \wp(\mathfrak{t})=-\mathbb{I}_{\mathrm{q}} \hbar(\mathfrak{t})+c_{1} \Gamma_{\mathrm{q}}(\varsigma)$. Therefore,

$$
\mathbb{D}_{\mathrm{q}}^{s-1} \wp(\infty)=-\int_{0}^{+\infty} \hbar(\xi) \mathrm{d}_{\mathrm{q}} \xi+c_{1} \Gamma_{\mathrm{q}}(\varsigma) .
$$

By the second condition of (7)

$$
\begin{aligned}
-\int_{0}^{+\infty} \hbar(\xi) \mathrm{d}_{\mathrm{q}} \xi+c_{1} \Gamma_{\mathrm{q}}(\varsigma)= & c_{1} \sum_{i=1}^{m}{ }_{1} \mathrm{a}_{i 1} \eta_{i}^{\varsigma-1} \\
& \left.-\frac{1}{\Gamma_{\mathrm{q}}(\varsigma)} \sum_{i=1}^{m}{ }_{1} \mathrm{a}_{i} \int_{0}^{1}{ }^{1 \eta_{i}}{ }_{1} \eta_{i}-\mathrm{q} \xi\right)^{(\varsigma-1)} \hbar(\xi) \mathrm{d}_{\mathrm{q}} \xi \\
& +c_{1} \sum_{i=1}^{m}{ }_{2} \mathrm{a}_{i} \frac{\Gamma(\varsigma)}{\Gamma(\varsigma-v)}{ }_{2} \eta_{i}^{\varsigma-v-1} \\
& \left.-\frac{1}{\Gamma_{\mathrm{q}}(\varsigma-v)} \sum_{i=1}^{m}{ }_{2} \mathrm{a}_{i} \int_{0}{ }^{2} \eta_{i}{ }_{2} \eta_{i}-\mathrm{q} \xi\right)^{(\varsigma-1)} \hbar(\xi) \mathrm{d}_{\mathrm{q}} \xi
\end{aligned}
$$

Moreover, equation (11) implies that

$$
\begin{aligned}
c_{1}= & \frac{1}{\Lambda} \int_{0}^{+\infty} \hbar(\xi) \mathrm{d}_{\mathrm{q}} \xi-\frac{1}{\Lambda} \sum_{i=1}^{m}{ }_{1} \mathrm{a}_{i} \int_{0}^{1 \eta_{i}} \frac{\left({ }_{1} \eta_{i}-\mathrm{q} \xi\right)^{(\varsigma-1)}}{\Gamma_{\mathrm{q}}(\varsigma)} \hbar(\xi) \mathrm{d}_{\mathrm{q}} \xi \\
& -\frac{1}{\Lambda} \sum_{i=1}^{m}{ }_{2} \mathrm{a}_{i} \int_{0}{ }_{0} \eta_{i} \frac{\left({ }_{2} \eta_{i}-\mathrm{q} \xi\right)^{(\varsigma-\nu-1)}}{\Gamma_{\mathrm{q}}(\varsigma-v)} \hbar(\xi) \mathrm{d}_{\mathrm{q}} \xi
\end{aligned}
$$

where $\Lambda$ is given by (9). Consequently, the unique solution of problem (6) and (7) is given by the following formula

$$
\begin{aligned}
\wp(\mathfrak{t})= & \frac{\mathfrak{t}^{\varsigma^{-1}}}{\Lambda} \int_{0}^{+\infty} \hbar(\xi) \mathrm{d}_{\mathrm{q}} \xi \\
& -\frac{\mathfrak{t}^{\varsigma-1}}{\Lambda} \sum_{i=1}^{m}{ }_{1} \mathrm{a}_{i} \int_{0}^{1 \eta_{i}} \frac{\left({ }_{1} \eta_{i}-\mathrm{q} \xi\right)^{(\varsigma-1)}}{\Gamma_{\mathrm{q}}(\varsigma)} \hbar(\xi) \mathrm{d}_{\mathrm{q}} \xi
\end{aligned}
$$


Boutiara et al. Journal of Inequalities and Applications

(2022) 2022:29

Page 8 of 27

$$
\begin{aligned}
& -\frac{\mathfrak{t}^{\varsigma-1}}{\Lambda} \sum_{i=1}^{m}{ }_{2} \mathrm{a}_{i} \int_{0}^{\eta_{i}} \frac{\left({ }_{2} \eta_{i}-\mathrm{q} \xi\right)^{(\varsigma-\nu-1)}}{\Gamma_{\mathrm{q}}(\varsigma-\nu)} \hbar(\xi) \mathrm{d}_{\mathrm{q}} \xi \\
& -\frac{1}{\Gamma_{\mathrm{q}}(\varsigma)} \int_{0}^{\mathrm{t}}(\mathfrak{t}-\mathrm{q} \xi)^{(\varsigma-1)} \hbar(\xi) \mathrm{d}_{\mathrm{q}} \xi \\
& =\frac{\mathfrak{t}^{\varsigma^{-1}} \Gamma_{\mathrm{q}}(\varsigma)}{\Lambda} \int_{0}^{+\infty} \frac{\hbar(\xi)}{\Gamma_{\mathrm{q}}(\varsigma)} \mathrm{d}_{\mathrm{q}} \xi \\
& -\frac{\mathfrak{t}^{5^{-1}}}{\Lambda} \sum_{i=1}^{m}{ }_{1} \mathrm{a}_{i} \int_{0}^{1 \eta_{i}} \frac{\left({ }_{1} \eta_{i}-\mathrm{q} \xi\right)^{(\varsigma-1)}}{\Gamma_{\mathrm{q}}(\varsigma)} \hbar(\xi) \mathrm{d}_{\mathrm{q}} \xi \\
& -\frac{\mathfrak{t}^{\varsigma-1}}{\Lambda} \sum_{i=1}^{m}{ }_{2} \mathrm{a}_{i} \int_{0}^{{ }_{2} \eta_{i}} \frac{\left({ }_{2} \eta_{i}-\mathrm{q} \xi\right)^{(\varsigma-v-1)}}{\Gamma_{\mathrm{q}}(\varsigma-v)} \hbar(\xi) \mathrm{d}_{\mathrm{q}} \xi \\
& -\frac{1}{\Gamma_{\mathrm{q}}(\varsigma)} \int_{0}^{\mathrm{t}}(\mathfrak{t}-\mathrm{q} \xi)^{(\varsigma-1)} \hbar(\xi) \mathrm{d}_{\mathrm{q}} \xi \\
& =\frac{1}{\Gamma_{\mathrm{q}}(\varsigma)-\sum_{i=1}^{m}{ }_{1} \mathrm{a}_{i 1} \eta_{i}-\sum_{i=1}^{m}{ }_{2} \mathrm{a}_{i} \frac{\Gamma(\varsigma)}{\Gamma(\varsigma-\nu)}{ }_{2} \eta_{i}^{\varsigma^{-\nu}-1}}\left[\left(\Gamma_{\mathrm{q}}(\varsigma)\right.\right. \\
& -\sum_{i=1}^{m}{ }_{1} \mathrm{a}_{i 1} \eta_{i}+\sum_{i=1}^{m}{ }_{1} \mathrm{a}_{i 1} \eta_{i}-\sum_{i=1}^{m}{ }_{2} \mathrm{a}_{i} \frac{\Gamma(\varsigma)}{\Gamma(\varsigma-v)}{ }_{2} \eta_{i}^{\varsigma-\nu-1} \\
& \left.\left.+\sum_{i=1}^{m}{ }_{2} \mathrm{a}_{i} \frac{\Gamma(\varsigma)}{\Gamma(\varsigma-v)}{ }_{2} \eta_{i}^{\varsigma-\nu-1}\right)\right] \frac{\mathfrak{t}^{\varsigma-1}}{\Gamma_{\mathrm{q}}(\varsigma)} \int_{0}^{+\infty} \hbar(\xi) \mathrm{d}_{\mathrm{q}} \xi \\
& -\frac{\mathfrak{t}^{\varsigma^{-1}}}{\Lambda} \sum_{i=1}^{m}{ }_{1} \mathrm{a}_{i} \int_{0}^{1 \eta_{i}} \frac{\left({ }_{1} \eta_{i}-\mathrm{q} \xi\right)^{(\varsigma-1)}}{\Gamma_{\mathrm{q}}(\varsigma)} \hbar(\xi) \mathrm{d}_{\mathrm{q}} \xi \\
& -\frac{\mathfrak{t}^{\varsigma-1}}{\Lambda} \sum_{i=1}^{m}{ }_{2} \mathrm{a}_{i} \int_{0}{ }^{2 \eta_{i}} \frac{\left({ }_{2} \eta_{i}-\mathrm{q} \xi\right)^{(\varsigma-\nu-1)}}{\Gamma_{\mathrm{q}}(\varsigma-v)} \hbar(\xi) \mathrm{d}_{\mathrm{q}} \xi \\
& -\frac{1}{\Gamma_{\mathrm{q}}(\varsigma)} \int_{0}^{\mathrm{t}}(\mathfrak{t}-\mathrm{q} \xi)^{(\varsigma-1)} \hbar(\xi) \mathrm{d}_{\mathrm{q}} \xi \\
& =\frac{1}{\Gamma_{\mathrm{q}}(\varsigma)} \int_{0}^{\mathfrak{t}}\left(\mathfrak{t}^{\zeta-1}-(\mathfrak{t}-\mathrm{q} \xi)^{(\varsigma-1)}\right) \hbar(\xi) \mathrm{d}_{\mathrm{q}} \xi \\
& +\frac{1}{\Gamma_{\mathrm{q}}(\varsigma)} \int_{\mathfrak{t}}^{+\infty} \mathfrak{t}^{\varsigma-1} \hbar(\xi) \mathrm{d}_{\mathrm{q}} \xi \\
& -\frac{\mathfrak{t}^{\varsigma-1}}{\Lambda \Gamma_{\mathrm{q}}(\varsigma)} \sum_{i=1}^{m}{ }_{1} \mathrm{a}_{i} \int_{0}^{1}{ }^{1} \eta_{i}\left({ }_{1} \eta_{i}^{\varsigma-1}-\left({ }_{1} \eta_{i}-\mathrm{q} \xi\right)^{(\varsigma-1)}\right) \hbar(\xi) \mathrm{d}_{\mathrm{q}} \xi \\
& +\frac{\mathfrak{t}^{5-1}}{\Lambda \Gamma_{\mathrm{q}}(\varsigma)} \sum_{i=1}^{m}{ }_{1} \mathrm{a}_{i} \int_{1 \eta_{i}}^{\infty}{ }_{1} \eta_{i}^{\varsigma^{-1}} \hbar(\xi) \mathrm{d}_{\mathrm{q}} \xi \\
& -\frac{\mathfrak{t}^{\varsigma-1}}{\Lambda \Gamma_{\mathrm{q}}(\varsigma-v)} \sum_{i=1}^{m}{ }_{2} \mathrm{a}_{i} \int_{0}^{2 \eta_{i}}\left({ }_{2} \eta_{i}^{\varsigma-\nu-1}-\left({ }_{2} \eta_{i}-\mathrm{q} \xi\right)^{(\varsigma-\nu-1)}\right) \hbar(\xi) \mathrm{d}_{\mathrm{q}} \xi \\
& +\frac{\mathfrak{t}^{\varsigma^{-1}}}{\Lambda \Gamma_{\mathrm{q}}(\varsigma-\nu)} \sum_{i=1}^{m}{ }_{2} \mathrm{a}_{i} \int_{2 \eta_{i}}^{\infty}{ }_{2} \eta_{i}^{\varsigma-\nu-1} \hbar(\xi) \mathrm{d}_{\mathrm{q}} \xi, \\
& =\int_{0}^{\infty}{ }_{1} \mathbb{G}_{\mathrm{q}}(\mathfrak{t}, \xi) \hbar(\xi) \mathrm{d}_{\mathrm{q}} \xi-\frac{\mathfrak{t}^{5-1}}{\Lambda} \sum_{i=1}^{m}{ }_{1} \mathrm{a}_{i} \int_{0}^{\infty}{ }_{2} \mathbb{G}_{\mathrm{q}}\left({ }_{1} \eta_{i}, \xi\right) \hbar(\xi) \mathrm{d}_{\mathrm{q}} \xi
\end{aligned}
$$




$$
\begin{aligned}
& -\frac{\mathfrak{t}^{\varsigma^{-1}}}{\Lambda} \sum_{i=1}^{m}{ }_{2} \mathrm{a}_{i} \int_{0}^{\infty}{ }_{2} \mathbb{G}_{\mathrm{q}}\left({ }_{2} \eta_{i}, \xi\right) \hbar(\xi) \mathrm{d}_{\mathrm{q}} \xi, \\
= & \int_{0}^{\infty}{ }_{1} \mathbb{G}_{\mathrm{q}}(\mathfrak{t}, \xi) \hbar(\xi) \mathrm{d}_{\mathrm{q}} \xi .
\end{aligned}
$$

The proof is completed.

Let us now mention some Green's function's properties that are essential to our study.

Lemma 2.6 Suppose that ${ }_{1} \mathbb{G}_{\mathrm{q}}(\mathfrak{t}, \mathfrak{s})$ and ${ }_{2} \mathbb{G}_{\mathrm{q}}(\mathfrak{t}, \mathfrak{s})$ are the Green's functions of the linear system (6) and (7) provided in Lemma 2.5. Then, we obtain:

(A1) $\mathbb{G}_{\mathrm{q}}(\mathfrak{t}, \mathfrak{s})$ is a continuous function for $(\mathfrak{t}, \mathfrak{s}) \in J^{2}$;

(A2) ${ }_{1} \mathbb{G}_{\mathrm{q}}(\mathrm{t}, \mathfrak{s}) \geq 0$ and ${ }_{1} \mathbb{G}_{\mathrm{q}}(\mathfrak{t}, \mathfrak{s}) \leq{ }_{1} \mathbb{G}_{\mathrm{q}}(\mathrm{q} \mathfrak{s}, \mathfrak{s}), \forall \mathfrak{t}, \mathfrak{s} \in J$.

(A3) for $(\mathfrak{t}, \mathfrak{s}) \in J^{2}$,

$$
\frac{{ }_{1} \mathbb{G}_{\mathrm{q}}(\mathfrak{t}, \mathfrak{s})}{1+\mathfrak{t}^{-1}} \leq \frac{1}{\Gamma_{\mathrm{q}}(\varsigma)}+\frac{\sum_{i=1}^{m} \mathrm{a}_{i 1} \eta_{i}^{\zeta-1}}{\Lambda \Gamma_{\mathrm{q}}(\varsigma)}+\frac{\sum_{i=1}^{m} \mathrm{a}_{i 2} \eta_{i}^{\zeta-v-1}}{\Lambda \Gamma_{\mathrm{q}}(\varsigma-v)}
$$

Proof Clearly, (A1) and (A2) hold. Obviously, ${ }_{1} \mathbb{G}_{\mathrm{q}}(\mathfrak{t}, \mathfrak{s}) \geq 0$, for $\mathfrak{t}, \mathfrak{s} \in J$. The monotonicity of ${ }_{1} \mathbb{G}_{\mathrm{q}}(\mathfrak{t}, \mathfrak{s})$ indicates

$$
\sup _{\mathfrak{t} \in J} \mathbb{G}_{\mathrm{q}}(\mathfrak{t}, \mathfrak{s})={ }_{1} \mathbb{G}_{\mathrm{q}}(\mathrm{q} \mathfrak{s}, \mathfrak{s})=\frac{(\mathrm{q} \mathfrak{s})^{\varsigma^{-1}}}{\Gamma_{\mathrm{q}}(\varsigma)}, \quad \forall \mathfrak{s} \in J
$$

To prove (A3), for $(\mathfrak{t q}, \mathfrak{t}) \in J^{2}$, we obtain

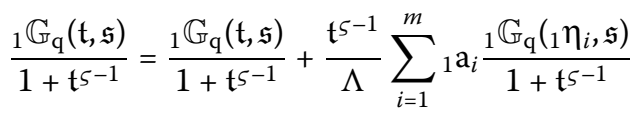

$$
\begin{aligned}
& +\frac{\mathfrak{t}^{\varsigma^{-1}}}{\Lambda} \sum_{i=1}^{m}{ }_{2} \mathrm{a}_{i} \frac{\mathbb{G}_{\mathrm{q}}\left({ }_{2} \eta_{i}, \mathfrak{s}\right)}{1+\mathfrak{t}^{5-1}} \\
& \leq\left(\frac{1}{\Gamma_{\mathrm{q}}(\varsigma)}+\frac{\sum_{i=1}^{m} \mathrm{a}_{i 1} \eta_{i}^{\zeta-1}}{\Lambda \Gamma_{\mathrm{q}}(\varsigma)}+\frac{\sum_{i=1}^{m} \mathrm{a}_{i 2} \eta_{i}^{\zeta-v-1}}{\Lambda \Gamma_{\mathrm{q}}(\varsigma-v)}\right)=: \nabla .
\end{aligned}
$$

The proof of Lemma 2.6 is completed.

\section{Main results}

First, we define the norm space

$$
\mathcal{C}_{\infty}(J, \mathbb{R})=\left\{\wp \in \mathcal{C}(J, \mathbb{R}): \lim _{\mathfrak{t} \rightarrow+\infty} \frac{\wp(\mathfrak{t})}{1+\mathfrak{t}^{5}-1} \text { exists }\right\}
$$

equipped with the norm

$$
\|\wp\|=\sup _{\mathfrak{t} \in J} \frac{|\wp(\mathfrak{t})|}{1+\mathfrak{t}^{5-1}} .
$$

The space $\mathcal{C}_{\infty}$ is a Banach space [7]. A map $\wp(\mathfrak{t}) \in \mathcal{C}(J, \mathbb{R})$ with its q-derivative of RiemannLiouville of order $\varsigma$ that exists on $J$ is named as a solution of (6) if (7) is satisfied. For 
$\wp \in \mathcal{C}_{\infty}$, let an operator $\mathfrak{H}$ be expressed as:

$$
\begin{aligned}
\mathfrak{H} \wp(\mathfrak{t})= & \int_{0}^{\infty}{ }_{1} \mathbb{G}_{\mathrm{q}}(\mathfrak{t}, \xi) \hbar(\xi) \mathrm{d}_{\mathrm{q}} \xi \\
& -\frac{\mathfrak{t}^{\varsigma-1}}{\Lambda} \sum_{i=1}^{m}{ }_{1} \mathrm{a}_{i} \int_{0}^{\infty}{ }_{2} \mathbb{G}_{\mathrm{q}}\left({ }_{1} \eta_{i}, \xi\right) \hbar(\xi) \mathrm{d}_{\mathrm{q}} \xi \\
& -\frac{\mathfrak{t}^{\varsigma-1}}{\Lambda} \sum_{i=1}^{m}{ }_{2} \mathrm{a}_{i} \int_{0}^{\infty}{ }_{2} \mathbb{G}_{\mathrm{q}}\left({ }_{2} \eta_{i}, \xi\right) \hbar(\xi) \mathrm{d}_{\mathrm{q}} \xi
\end{aligned}
$$

Clearly, we have a continuous, $\wp \in \mathcal{C}_{\infty}(J, \mathbb{R})$, then $\wp$ is the problem (4) solution if $\wp(\mathfrak{t})=$ $\mathfrak{H} \wp(\mathfrak{t})$ for any $\mathfrak{t} \in \mathbb{R}$. The operator $\mathfrak{H}$ compactness is shown by proving that $\mathfrak{H}$ has a fixed point on $\mathcal{C}_{\infty}(J, \mathbb{R})$.

Remark 3.1 Note that to apply the Arzelá-Ascoli theorem in basic space $\mathcal{C}_{\infty}$, we need to establish the following modified compactness criterion to show that $\mathfrak{H}$ is compact.

Let us introduce the following hypotheses for convenience:

(A4) for each $\mathrm{q} \in(0,1)$,

$$
0 \leq\left\{\sum_{i=1}^{m}{ }_{1} \mathrm{a}_{i 1} \eta_{i}^{\varsigma-1}+\sum_{i=1}^{m}{ }_{2} \mathrm{a}_{i} \frac{\Gamma(\varsigma)}{\Gamma(\varsigma-v)}{ }_{2} \eta_{i}^{\varsigma-v-1}\right\}<\Gamma_{\mathrm{q}}(\varsigma)
$$

(A5) Let

$$
\mathfrak{w}_{*}(\mathfrak{t}, \wp)=\mathfrak{w}\left(\mathfrak{t},\left(1+\mathfrak{t}^{\varsigma-1}\right) \wp\right)
$$

and $\left|\mathfrak{w}_{*}(\mathfrak{t}, \wp)\right| \leq \psi(\mathfrak{t}) \mathfrak{p}(|\wp|)$, on $J$ with nondecreasing function $\mathfrak{p} \in \mathcal{C}(J, \mathbb{R})$ and $\psi \in \mathcal{C}(\jmath)$,

$$
\int_{0}^{\infty} \psi(\xi) \mathrm{d}_{\mathrm{q}} \xi<+\infty
$$

(A6) $\exists$ is a constant $\gamma$, for any $\xi \in J, \wp, \wp \in \mathbb{R}$

$$
\left|\mathfrak{w}_{*}(\xi, \wp(\xi))-\mathfrak{w}_{*}(\xi, \wp(\xi))\right| \leq \gamma|\wp-\wp|
$$

Lemma 3.1 ([7]) Consider the two sets $\Upsilon=\left\{\wp \in \mathcal{C}_{\infty}:\|\wp\|<\mu\right\}(\mu>0)$, and

$$
\hat{\Upsilon}=\left\{\frac{\wp(\mathfrak{t})}{1+\mathfrak{t}^{-1}}: \wp \in \Upsilon\right\}
$$

If $\hat{\Upsilon}$ is equicontinuous on any compact intervals of $\mathrm{J}$ and equiconvergent at $\infty$, then $\Upsilon$ is relatively compact on $\mathcal{C}_{\infty}$

Remark $3.2 \hat{\Upsilon}$ is termed equiconvergent at $\infty$ iff, for any given positive number $\epsilon, \exists \mathrm{N}=$ $\mathrm{N}_{\epsilon}>0$,

$$
\left|\frac{\wp(\mathfrak{t})}{1+\mathfrak{t} \varsigma^{-1}}-\frac{\wp(\mathfrak{t})}{1+\mathfrak{t}^{-1}}\right|<\varepsilon \quad \forall \wp \in \hat{\Upsilon}, \mathfrak{t}, \mathfrak{t} \geq N
$$


Lemma 3.2 A cone $\mathbb{P} \subset \mathcal{C}_{\infty}$ is defined by

$$
\mathbb{P}=\left\{\wp \in \mathcal{C}_{\infty}: \wp(\mathfrak{t}) \geq 0, \mathfrak{t} \in J\right\}
$$

If $(A 4)$ and $(A 5)$ hold, then $\mathfrak{H}: \mathbb{P} \rightarrow \mathbb{P}$ is completely continuous.

Proof This proof consists of 3 essential steps.

STEP A: Let us prove that $\mathfrak{H}: \mathbb{P} \rightarrow \mathbb{P}$ is continuous. From the continuity and nonnegativity of ${ }_{1} \mathcal{G}_{\mathrm{q}}$ and $\mathfrak{w}$, we obtain $\mathfrak{H} \wp(\mathfrak{t}) \geq 0$ for $\mathfrak{t} \in J$. For each $\wp \in \mathbb{P}$, by (A4), we obtain:

$$
\int_{0}^{\infty} \mathfrak{w}(\xi, \wp(\xi)) \mathrm{d}_{\mathrm{q}} \xi \leq \mathfrak{p}(\mu) \int_{0}^{\infty} \psi(\xi) \mathrm{d}_{\mathrm{q}} \xi<\infty,
$$

and

$$
\begin{aligned}
\lim _{\mathfrak{t} \rightarrow+\infty} \frac{(\mathfrak{H} \wp)(\mathfrak{t})}{1+\mathfrak{t}^{5-1}}= & \int_{0}^{\infty}{ }_{1} \mathbb{G}_{\mathrm{q}}(\mathfrak{t}, \xi) \mathfrak{w}(\xi, \wp(\xi)) \mathrm{d}_{\mathrm{q}} \xi \\
& -\frac{\mathfrak{t}^{5-1}}{\Lambda} \sum_{i=1}^{m}{ }_{1} \mathrm{a}_{i} \int_{0}^{\infty}{ }_{2} \mathbb{G}_{\mathrm{q}}\left({ }_{1} \eta_{i}, \xi\right) \mathfrak{w}(\xi, \wp(\xi)) \mathrm{d}_{\mathrm{q}} \xi \\
& -\frac{\mathfrak{t}^{5}-1}{\Lambda} \sum_{i=1}^{m}{ }_{2} \mathrm{a}_{i} \int_{0}^{\infty}{ }_{2} \mathbb{G}_{\mathrm{q}}\left({ }_{2} \eta_{i}, \xi\right) \mathfrak{w}(\xi, \wp(\xi)) \mathrm{d}_{\mathrm{q}} \xi
\end{aligned}
$$

Thus,

$$
\lim _{\mathfrak{t} \rightarrow+\infty} \frac{(\mathfrak{H} \wp)(\mathfrak{t})}{1+\mathfrak{t}^{-1}}
$$

exists. Thus, $\mathfrak{H}(\mathbb{P}) \subset \mathbb{P}$. Let $\wp_{1}(\mathfrak{t})=\frac{\wp(\mathfrak{t})}{1+\mathfrak{t}^{-1}}$ and

$$
\wp_{n 1}(\mathfrak{t})=\frac{\wp_{n}(\mathfrak{t})}{1+\mathfrak{t}^{5-1}} .
$$

Then by (A4),

$$
\mathfrak{w}(\xi, \wp(\xi))=\mathfrak{w}_{*}\left(\xi, \frac{\wp_{n}(\xi)}{1+\xi^{\varsigma^{-1}}}\right)=\mathfrak{w}_{*}\left(\xi, \wp_{1}(\xi)\right) .
$$

Taking $\wp_{n} \rightarrow \wp$ as $n \rightarrow+\infty$ in $\mathcal{C}_{\infty}$, by (A5), we obtain:

$$
\begin{aligned}
\left|\mathfrak{w}\left(\mathfrak{t}, \wp_{n}(\mathfrak{t})\right)-\mathfrak{w}(\mathfrak{t}, \wp(\mathfrak{t}))\right| & =\left|\mathfrak{w}_{*}\left(\mathfrak{t}, \frac{\wp_{n}(\mathfrak{t})}{1+\mathfrak{t}^{5}-1}\right)-\mathfrak{w}_{*}\left(\mathfrak{t}, \frac{\wp(\mathfrak{t})}{1+\mathfrak{t}^{5-1}}\right)\right| \\
& =\left|\mathfrak{w}_{*}\left(\mathfrak{t}, \wp_{1_{n}}(\mathfrak{t})\right)-\mathfrak{w}_{*}\left(\mathfrak{t}, \wp_{1}(\mathfrak{t})\right)\right| \\
& \leq \gamma\left|\wp_{1_{n}}(\mathfrak{t})-\wp_{1}(\mathfrak{t})\right| \rightarrow 0 \quad \text { uniformly on } J .
\end{aligned}
$$

So $\mathfrak{w}\left(\mathfrak{t}, \wp_{n}(\mathfrak{t})\right) \rightarrow \mathfrak{w}(\mathfrak{t}, \wp(\mathfrak{t}))$ uniformly on $J$. By Lemma 2.4, we obtain

$$
\lim _{n \rightarrow \infty} \int_{0}^{\infty} \mathfrak{w}\left(\xi, \wp_{n}(\xi)\right) d_{\mathrm{q}} \xi=\int_{0}^{\infty} \mathfrak{w}(\xi, \wp(\xi)) \mathrm{d}_{\mathrm{q}} \xi .
$$


Hence, combining (15), we obtain

$$
\begin{aligned}
& \left|\frac{\mathfrak{H} \wp_{n}(\mathfrak{t})}{1+\varsigma^{-1}}-\frac{\mathfrak{H} \wp(\mathfrak{t})}{1+\mathfrak{t} \varsigma^{-1}}\right| \\
& =\mid \int_{0}^{\infty} \frac{1 \mathbb{G}_{\mathrm{q}}(\mathfrak{t}, \xi)}{1+\mathfrak{t}^{-1}}\left(\mathfrak{w}\left(\xi, \wp_{n}(\xi)\right)-\mathfrak{w}(\xi, \wp(\xi))\right) \mathrm{d}_{\mathrm{q}} \xi \\
& +\frac{\mathfrak{t}^{\varsigma-1}}{\Lambda} \sum_{i=1}^{m}{ }_{1} \mathrm{a}_{i} \int_{0}^{\infty} \frac{{ }_{2} \mathbb{G}_{\mathrm{q}}\left({ }_{1} \eta_{i}, \xi\right)}{1+\mathfrak{t}^{-1}}\left(\mathfrak{w}\left(\xi, \wp_{n}(\xi)\right)-\mathfrak{w}(\xi, \wp(\xi))\right) \mathrm{d}_{\mathrm{q}} \xi \\
& +\frac{\mathfrak{t}^{\varsigma^{-1}}}{\Lambda} \sum_{i=1}^{m}{ }_{2} \mathrm{a}_{i} \int_{0}^{\infty} \frac{{ }_{2} \mathbb{G}_{\mathrm{q}}\left({ }_{2} \eta_{i}, \xi\right)}{1+\mathfrak{t}^{-1}}\left(\mathfrak{w}\left(\xi, \wp_{n}(\xi)\right)-\mathfrak{w}(\xi, \wp(\xi))\right) \mathrm{d}_{\mathrm{q}} \xi \\
& \leq\left|\int_{0}^{\infty} \frac{{ }_{1} \mathbb{G}_{\mathrm{q}}(\mathfrak{t}, \xi)}{1+\mathfrak{t}^{-1}}\left(\mathfrak{w}\left(\xi, \wp_{n}(\xi)\right)-\mathfrak{w}(\xi, \wp(\xi))\right) \mathrm{d}_{\mathrm{q}} \xi\right| \\
& +\left|\frac{\mathfrak{t}^{S^{-1}}}{\Lambda} \sum_{i=1}^{m}{ }_{1} \mathrm{a}_{i} \int_{0}^{\infty} \frac{{ }_{2} \mathbb{G}_{\mathrm{q}}\left({ }_{1} \eta_{i}, \xi\right)}{1+\mathfrak{t}^{-1}}\left(\mathfrak{w}\left(\xi, \wp_{n}(\xi)\right)-\mathfrak{w}(\xi, \wp(\xi))\right) \mathrm{d}_{\mathrm{q}} \xi\right| \\
& +\left|\frac{\mathfrak{t}^{\varsigma-1}}{\Lambda} \sum_{i=1}^{m}{ }_{2} \mathrm{a}_{i} \int_{0}^{\infty} \frac{{ }_{2} \mathbb{G}_{\mathrm{q}}\left({ }_{2} \eta_{i}, \xi\right)}{1+\mathfrak{t}^{5}-1}\left(\mathfrak{w}\left(\xi, \wp_{n}(\xi)\right)-\mathfrak{w}(\xi, \wp(\xi))\right) \mathrm{d}_{\mathrm{q}} \xi\right| \\
& \leq \frac{1}{\Gamma_{\mathrm{q}}(\varsigma)}\left|\int_{0}^{\infty}\left(\mathfrak{w}\left(\xi, \wp_{n}(\xi)\right)-\mathfrak{w}(\xi, \wp(\xi))\right) \mathrm{d}_{\mathrm{q}} \xi\right| \\
& +\left|\frac{\sum_{i=11}^{m} \mathrm{a}_{i 1} \eta_{i}^{\zeta-1}}{\Lambda \Gamma_{\mathrm{q}}(\varsigma)} \int_{0}^{\infty}\left(\mathfrak{w}\left(\xi, \wp_{n}(\xi)\right)-\mathfrak{w}(\xi, \wp(\xi))\right) \mathrm{d}_{\mathrm{q}} \xi\right| \\
& +\left|\frac{\sum_{i=12}^{m} \mathrm{a}_{i 2} \eta_{i}^{\varsigma-\nu-1}}{\Lambda \Gamma_{\mathrm{q}}(\varsigma-v)} \int_{0}^{\infty}\left(\mathfrak{w}\left(\xi, \wp_{n}(\xi)\right)-\mathfrak{w}(\xi, \wp(\xi))\right) \mathrm{d}_{\mathrm{q}} \xi\right| .
\end{aligned}
$$

Hence,

$$
\left\|\mathfrak{H} \wp_{n}-\mathfrak{H} \wp\right\|=\sup _{\mathfrak{t} \in J}\left|\frac{\mathfrak{H} \wp_{n}(\mathfrak{t})-\mathfrak{H} \wp(\mathfrak{t})}{1+\mathfrak{t}^{-1}}\right| \rightarrow 0
$$

as $n \rightarrow \infty$. So, $\mathfrak{H}$ is continuous.

STEP B: We show that $\mathfrak{H}$ is uniformly bounded. For this case, let $\mathbb{Q}$ be any bounded subset of $\mathbb{P}$, i.e., $\exists \mu>0,\|\wp\| \leq \mu$ for any $\wp \in \mathbb{Q}$. It is enough to prove that $\mathfrak{H}(\wp)$ is bounded in $\mathbb{P}$. For $\wp \in \mathbb{Q}$, we obtain:

$$
\begin{aligned}
\|\mathfrak{H} \wp\|= & \sup _{\mathfrak{t} \in J}\left(\int_{0}^{\infty} \frac{{ }_{1} \mathbb{G}_{\mathrm{q}}(\mathfrak{t}, \xi)}{1+\mathfrak{t}^{5-1}} \mathfrak{w}(\mathfrak{t}, \wp(\xi)) \mathrm{d}_{\mathrm{q}} \xi\right. \\
& +\frac{\mathfrak{t}^{\varsigma-1}}{\Lambda} \sum_{i=1}^{m}{ }_{1} \mathrm{a}_{i} \int_{0}^{\infty} \frac{{ }_{2} \mathbb{G}_{\mathrm{q}}\left(\xi_{i}, \xi\right)}{1+\mathfrak{t}^{-1}} \mathfrak{w}(\mathfrak{t}, \wp(\xi)) \mathrm{d}_{\mathrm{q}} \xi \\
& \left.+\frac{\mathfrak{t}^{\varsigma^{-1}}}{\Lambda} \sum_{i=1}^{m}{ }_{2} \mathrm{a}_{i} \int_{0}^{\infty} \frac{{ }_{2} \mathbb{G}_{\mathrm{q}}\left({ }_{2} \eta_{i}, \xi\right)}{1+\mathfrak{t}^{-1}} \mathfrak{w}(\mathfrak{t}, \wp(\xi)) \mathrm{d}_{\mathrm{q}} \xi\right) \\
\leq & \frac{1}{\Gamma_{\mathrm{q}}(\varsigma)} \int_{0}^{\infty}|\mathfrak{w}(\mathfrak{t}, \wp(\xi))| \mathrm{d}_{\mathrm{q}} \xi
\end{aligned}
$$




$$
\begin{aligned}
& +\frac{\sum_{i=11}^{m} \mathrm{a}_{i 1} \eta_{i}^{\varsigma-1}}{\Lambda \Gamma_{\mathrm{q}}(\varsigma)} \int_{0}^{\infty}|\mathfrak{w}(\mathfrak{t}, \wp(\xi))| \mathrm{d}_{\mathrm{q}} \xi \\
& +\frac{\sum_{i=1}^{m}{ }_{2} \mathrm{a}_{i 2} \eta_{i}^{\varsigma-\nu-1}}{\Lambda \Gamma_{\mathrm{q}}(\varsigma-v)} \int_{0}^{\infty} \mathfrak{w}(\mathfrak{t}, \wp(\xi)) \mathrm{d}_{\mathrm{q}} \xi \\
& =\left(\frac{1}{\Gamma_{\mathrm{q}}(\varsigma)}+\frac{\sum_{i=1}^{m} \mathrm{a}_{i 1} \eta_{i}^{\varsigma-1}}{\Lambda \Gamma_{\mathrm{q}}(\varsigma)}\right. \\
& \left.+\frac{\sum_{i=1}^{m} 2 \mathrm{a}_{i 2} \eta_{i}^{\zeta-\nu-1}}{\Lambda \Gamma_{\mathrm{q}}(\varsigma-v)}\right) \int_{0}^{\infty}\left|\mathfrak{w}\left(\xi, \frac{\wp(\xi)\left(1+\xi^{\varsigma^{-1}}\right)}{1+\xi^{\varsigma^{-1}}}\right)\right| \mathrm{d}_{\mathrm{q}} \xi \\
& =\left(\frac{1}{\Gamma_{\mathrm{q}}(\varsigma)}+\frac{\sum_{i=1}^{m} \mathrm{a}_{i 1} \eta_{i}^{\varsigma-1}}{\Lambda \Gamma_{\mathrm{q}}(\varsigma)}\right. \\
& \left.+\frac{\sum_{i=1}^{m}{ }_{2} \mathrm{a}_{i 2} \eta_{i}^{\varsigma-v-1}}{\Lambda \Gamma_{\mathrm{q}}(\varsigma-v)}\right) \int_{0}^{\infty}\left|\mathfrak{w}\left(\xi, \frac{\wp(\xi)}{1+\xi^{\varsigma-1}}\right)\right| \mathrm{d}_{\mathrm{q}} \xi \\
& \leq \mathfrak{p}(\mu) \nabla \int_{0}^{\infty} \psi(\xi) \mathrm{d}_{\mathrm{q}} \xi<\infty \text {. }
\end{aligned}
$$

Hence, $\mathfrak{H}(\mathbb{Q})$ is uniformly bounded. Now, we show that $\mathfrak{H}(\mathbb{Q})$ is equicontinuous on any compact interval. First, for each given $\bar{s}>0, \mathfrak{t}_{1}, \mathfrak{t}_{2} \in[0, \bar{s}]$, and $\wp \in \mathbb{P}$, and $\mathfrak{t}_{2}>\mathfrak{t}_{1}$, we deduce:

$$
\begin{aligned}
& \left|\frac{\mathfrak{H} \wp\left(\mathfrak{t}_{2}\right)}{1+\mathfrak{t}_{2}^{\zeta-1}}-\frac{\mathfrak{H} \wp\left(\mathfrak{t}_{1}\right)}{1+\mathfrak{t}_{1}^{\zeta-1}}\right| \leq \mid \int_{0}^{\infty} \frac{{ }_{1} \mathbb{G}_{\mathrm{q}}\left(\mathfrak{t}_{2}, \xi\right)}{1+\mathfrak{t}_{2}^{\zeta-1}} \mathfrak{w}(\xi, \wp(\xi)) \mathrm{d}_{\mathrm{q}} \xi \\
& -\int_{0}^{\infty} \frac{{ }_{1} \mathbb{G}_{\mathrm{q}}\left(\mathfrak{t}_{1}, \xi\right)}{1+\mathfrak{t}_{1}^{\varsigma-1}} \mathfrak{w}(\xi, \wp(\xi)) \mathrm{d}_{\mathrm{q}} \xi \\
& +\mid\left(\frac{\mathfrak{t}_{2}^{\varsigma-1}}{1+\mathfrak{t}_{2}^{\mathfrak{S}^{-1}}}-\frac{\mathfrak{t}_{1}^{\varsigma^{-1}}}{1+\mathfrak{t}_{1}^{\varsigma-1}}\right) \\
& \times \sum_{i=1}^{m}{ }_{1} \mathrm{a}_{i} \int_{0}^{\infty} \frac{{ }_{2} \mathbb{G}_{\mathbf{q}}\left({ }_{1} \eta_{i}, \xi\right)}{\Lambda} \mathfrak{w}(\xi, \wp(\xi)) \mathrm{d}_{\mathrm{q}} \xi \\
& +\mid\left(\frac{\mathfrak{t}_{2}^{\varsigma-1}}{1+\mathfrak{t}_{2}^{\zeta-1}}-\frac{\mathfrak{t}_{1}^{\varsigma-1}}{1+\mathfrak{t}_{1}^{\varsigma-1}}\right) \\
& \times \sum_{i=1}^{m}{ }_{2} \mathrm{a}_{i} \int_{0}^{\infty} \frac{{ }_{2} \mathbb{G}_{\mathrm{q}}\left({ }_{2} \eta_{i}, \xi\right)}{\Lambda} \mathfrak{w}(\xi, \wp(\xi)) \mathrm{d}_{\mathrm{q}} \xi \\
& \leq \mid \int_{0}^{\infty} \frac{{ }_{1} \mathbb{G}_{\mathrm{q}}\left(\mathfrak{t}_{2}, \xi\right)}{1+\mathfrak{t}_{2}^{\varsigma-1}} \mathfrak{w}(\xi, \wp(\xi)) \mathrm{d}_{\mathrm{q}} \xi \\
& -\int_{0}^{\infty} \frac{{ }_{1} \mathbb{G}_{\mathrm{q}}\left(\mathfrak{t}_{1}, \xi\right)}{1+\mathfrak{t}_{2}^{\varsigma-1}} \mathfrak{w}(\xi, \wp(\xi)) \mathrm{d}_{\mathrm{q}} \xi \\
& +\mid \int_{0}^{\infty} \frac{{ }_{1} \mathbb{G}_{\mathrm{q}}\left(\mathfrak{t}_{1}, \xi\right)}{1+\mathfrak{t}_{2}^{\varsigma-1}} \mathfrak{w}(\xi, \wp(\xi)) \mathrm{d}_{\mathrm{q}} \xi \\
& -\int_{0}^{\infty} \frac{{ }_{1} \mathbb{G}_{\mathrm{q}}\left(\mathfrak{t}_{1}, \xi\right)}{1+\mathfrak{t}_{1}^{\varsigma-1}} \mathfrak{w}(\xi, \wp(\xi)) \mathrm{d}_{\mathrm{q}} \xi \\
& +\mid\left(\frac{\mathfrak{t}_{2}^{\zeta-1}}{1+\mathfrak{t}_{2}^{\mathfrak{S}^{-1}}}-\frac{\mathfrak{t}_{1}^{\varsigma^{-1}}}{1+\mathfrak{t}_{1}^{\varsigma-1}}\right)
\end{aligned}
$$




$$
\begin{aligned}
& \times \sum_{i=1}^{m}{ }_{1} \mathrm{a}_{i} \int_{0}^{\infty} \frac{{ }_{2} \mathbb{G}_{\mathrm{q}}\left({ }_{1} \eta_{i}, \xi\right)}{\Gamma_{\mathrm{q}}(\varsigma)-\Lambda} \mathfrak{w}(\xi, \wp(\xi)) \mathrm{d}_{\mathrm{q}} \xi \mid \\
& +\mid\left(\frac{\mathfrak{t}_{2}^{\zeta-1}}{1+\mathfrak{t}_{2}^{\zeta-1}}-\frac{\mathfrak{t}_{1}^{\zeta-1}}{1+\mathfrak{t}_{1}^{\zeta-1}}\right) \\
& \times \sum_{i=1}^{m}{ }_{2} \mathrm{a}_{i} \int_{0}^{\infty} \frac{{ }_{2} \mathbb{G}_{\mathrm{q}}\left({ }_{2} \eta_{i}, \xi\right)}{\Lambda} \mathfrak{w}(\xi, \wp(\xi)) \mathrm{d}_{\mathrm{q}} \xi \mid .
\end{aligned}
$$

On the other hand, for all $\wp \in \mathbb{Q}, \mathfrak{t}_{1} \rightarrow \mathfrak{t}_{2}$, we obtain

$$
\begin{aligned}
& \int_{0}^{\infty} \frac{{ }_{1} \mathbb{G}_{\mathrm{q}}\left(\mathfrak{t}_{2}, \xi\right)-{ }_{1} \mathbb{G}_{\mathrm{q}}\left(\mathfrak{t}_{1}, \xi\right)}{1+\mathfrak{t}_{2}^{\zeta-1}}|\mathfrak{w}(\xi, \wp(\xi))| \mathrm{d}_{\mathrm{q}} \xi \\
& \leq \int_{0}^{\mathfrak{t}_{1}}\left|\frac{\left(\mathfrak{t}_{2}^{\varsigma^{-1}}-\mathfrak{t}_{1}^{\mathfrak{s}^{-1}}\right)-\left(\left(\mathfrak{t}_{2}-\mathrm{q} \xi\right)^{(\varsigma-1)}-\left(\mathfrak{t}_{1}-\mathrm{q} \xi\right)^{(\varsigma-1)}\right)}{\Gamma_{\mathrm{q}}(\varsigma)\left(1+\mathfrak{t}_{2}^{\varsigma-1}\right)}\right| \mathfrak{w}(\xi, \wp(\xi)) \mathrm{d}_{\mathrm{q}} \xi \\
& +\int_{\mathfrak{t}_{1}}^{\mathfrak{t}_{2}}\left|\frac{\mathfrak{t}_{2}^{\varsigma-1}-\mathfrak{t}_{1}^{\varsigma-1}-\left(\mathfrak{t}_{2}-\mathrm{q} \xi\right)^{(\varsigma-1)}}{\Gamma_{\mathrm{q}}(\varsigma)\left(1+\mathfrak{t}_{2}^{\varsigma-1}\right)}\right| \mathfrak{w}(\xi, \wp(\xi)) \mathrm{d}_{\mathrm{q}} \xi \\
& +\int_{\mathfrak{t}_{2}}^{\infty}\left|\frac{\mathfrak{t}_{2}^{\varsigma-1}-\mathfrak{t}_{1}^{\varsigma-1}}{\Gamma_{\mathrm{q}}(\varsigma)\left(1+\mathfrak{t}_{2}^{\varsigma-1}\right)}\right| \mathfrak{w}(\xi, \wp(\xi)) \mathrm{d}_{\mathrm{q}} \xi \\
& \leq \int_{0}^{\mathfrak{t}_{1}} \frac{\left(\mathfrak{t}_{2}^{\varsigma-1}-\mathfrak{t}_{1}^{\varsigma-1}\right)+\left(\left(\mathfrak{t}_{2}-\mathrm{q} \xi\right)^{(\varsigma-1)}-\left(\mathfrak{t}_{1}-\mathrm{q}\right)^{(\varsigma-1)}\right)}{\Gamma_{\mathrm{q}}(\varsigma)\left(1+\mathfrak{t}_{2}^{\zeta-1}\right)} \mathfrak{w}(\xi, \wp(\xi)) \mathrm{d}_{\mathrm{q}} \xi \\
& +\frac{3 \bar{s}^{\varsigma-1}}{\Gamma_{\mathrm{q}}(\varsigma)\left(1+\mathfrak{t}_{2}^{\varsigma-1}\right)} \int_{\mathfrak{t}_{1}}^{\mathfrak{t}_{2}} \mathfrak{w}(\xi, \wp(\xi)) \mathrm{d}_{\mathrm{q}} \xi \\
& +\frac{\mathfrak{t}_{2}^{\varsigma-1}-\mathfrak{t}_{1}^{\mathfrak{s}^{-1}}}{\Gamma_{\mathrm{q}}(\varsigma)\left(1+\mathfrak{t}_{2}^{\varsigma-1}\right)} \int_{\mathfrak{t}_{2}}^{\infty} \mathfrak{w}(\xi, \wp(\xi)) \mathrm{d}_{\mathrm{q}} \xi \\
& \leq \mathfrak{p}(\mu) \int_{0}^{\mathfrak{t}_{1}} \frac{\left(\mathfrak{t}_{2}^{\zeta-1}-\mathfrak{t}_{1}^{\varsigma-1}\right)+\left(\left(\mathfrak{t}_{2}-\mathrm{q} \xi\right)^{(\varsigma-1)}-\left(\mathfrak{t}_{1}-\mathrm{q} \xi\right)^{(\varsigma-1)}\right)}{\Gamma_{\mathrm{q}}(\varsigma)\left(1+\mathfrak{t}_{2}^{\zeta-1}\right)} \psi(\xi) \mathrm{d}_{\mathrm{q}} \xi \\
& +\mathfrak{p}(\mu) \frac{3 \bar{s}^{\zeta-1}}{\Gamma_{\mathrm{q}}(\varsigma)\left(1+\mathfrak{t}_{2}^{\zeta-1}\right)} \int_{\mathfrak{t}_{1}}^{\mathfrak{t}_{2}} \psi(\xi) \mathrm{d}_{\mathrm{q}} \xi \\
& +\mathfrak{p}(\mu) \frac{\mathfrak{t}_{2}^{\varsigma-1}-\mathfrak{t}_{1}^{\varsigma-1}}{\Gamma_{\mathrm{q}}(\varsigma)\left(1+\mathfrak{t}_{2}^{\varsigma-1}\right)} \int_{\mathfrak{t}_{2}}^{\infty} \psi(\xi) \mathrm{d}_{\mathrm{q}} \xi \rightarrow 0 .
\end{aligned}
$$

Similar to (16), for all $\wp \in \mathbb{Q}$, $\mathfrak{t}_{1} \rightarrow \mathfrak{t}_{2}$, we obtain

$$
\begin{aligned}
& \left|\int_{0}^{\infty} \frac{{ }_{1} \mathbb{G}_{\mathbf{q}}\left(\mathfrak{t}_{1}, \xi\right)}{1+\mathfrak{t}_{2}^{\sigma-1}} \mathfrak{w}(\xi, \wp(\xi)) \mathrm{d}_{\mathrm{q}} \xi-\int_{0}^{\infty} \frac{{ }_{1} \mathbb{G}_{\mathrm{q}}\left(\mathfrak{t}_{1}, \xi\right)}{1+\mathfrak{t}_{1}^{\zeta-1}} \mathfrak{w}(\xi, \wp(\xi)) \mathrm{d}_{\mathrm{q}} \xi\right| \\
& \leq \int_{0}^{\infty} \frac{{ }_{1} \mathbb{G}_{\mathrm{q}}(\mathfrak{t}, \xi)\left|\left(1+\mathfrak{t}_{1}^{\zeta-1}\right)-\left(1+\mathfrak{t}_{2}^{\zeta-1}\right)\right|}{\left(1+\mathfrak{t}_{1}^{\zeta-1}\right)\left(1+\mathfrak{t}_{2}^{\zeta-1}\right)} \mathfrak{w}(\xi, \wp(\xi)) \mathrm{d}_{\mathrm{q}} \xi \\
& \leq \frac{\left|\mathfrak{t}_{2}^{\zeta-1}-\mathfrak{t}_{1}^{\zeta-1}\right|}{\left(1+\mathfrak{t}_{2}^{\mathfrak{s}^{-1}}\right)} \int_{0}^{\infty} \frac{1 \mathbb{G}_{\mathbf{q}}\left(\mathfrak{t}_{1}, \xi\right)}{1+\mathfrak{t}_{1}^{\zeta-1} \mathfrak{w}(\xi, \wp(\xi)) \mathrm{d}_{\mathrm{q}} \xi} \\
& \leq \frac{\left|\mathfrak{t}_{2}^{\varsigma-1}-\mathfrak{t}_{1}^{\varsigma-1}\right|}{\left(1+\mathfrak{t}_{2}^{\varsigma-1}\right)} \int_{0}^{\infty} \frac{1}{\Gamma_{\mathrm{q}}(\varsigma)} \mathfrak{p}(\mu) \psi(\xi) \mathrm{d}_{\mathrm{q}} \xi
\end{aligned}
$$




$$
+\mathfrak{p}(\mu) \frac{\left|\mathfrak{t}_{2}^{\varsigma-1}-\mathfrak{t}_{1}^{\varsigma-1}\right|}{\Gamma_{\mathrm{q}}(\varsigma)\left(1+\mathfrak{t}_{2}^{\varsigma-1}\right)} \int_{0}^{\infty} \psi(\xi) \mathrm{d}_{\mathrm{q}} \xi \rightarrow 0,
$$

and

$$
\begin{aligned}
& \mid\left(\frac{\mathfrak{t}_{2}^{\zeta-1}}{1+\mathfrak{t}_{2}^{\zeta-1}}-\frac{\mathfrak{t}_{1}^{\zeta-1}}{1+\mathfrak{t}_{1}^{\zeta-1}}\right)\left(\sum_{i=1}^{m}{ }_{1} \mathrm{a}_{i} \int_{0}^{\infty} \frac{{ }_{2} \mathbb{G}_{\mathrm{q}}\left({ }_{1} \eta_{i}, \xi\right)}{\Lambda} \mathfrak{w}(\xi, \wp(\xi)) \mathrm{d}_{\mathrm{q}} \xi\right. \\
& \left.+\sum_{i=1}^{m}{ }_{2} \mathrm{a}_{i} \int_{0}^{\infty} \frac{{ }_{2} \mathbb{G}_{\mathrm{q}}\left({ }_{2} \eta_{i}, \xi\right)}{\Lambda} \mathfrak{w}(\xi, \wp(\xi)) \mathrm{d}_{\mathrm{q}} \xi\right) \\
& \leq \frac{\left|\mathfrak{t}_{2}^{\varsigma-1}-\mathfrak{t}_{1}^{\varsigma-1}\right|}{\left(1+\mathfrak{t}_{2}^{\zeta-1}\right)\left(1+\mathfrak{t}_{1}^{\varsigma-1}\right)} \int_{0}^{\infty}\left(\frac{\sum_{i=1}^{m} \mathrm{a}_{i 1} \eta_{i}^{\zeta-1}}{\Lambda \Gamma_{\mathrm{q}}(\varsigma)}\right. \\
& \left.+\frac{\sum_{i=1}^{m}{ }_{2} \mathrm{a}_{i 2} \eta_{i}^{\zeta-v-1}}{\Lambda \Gamma_{\mathrm{q}}(\varsigma-v)}\right) \mathfrak{w}(\xi, \wp(\xi)) \mathrm{d}_{\mathrm{q}} \xi \\
& \leq \frac{\left|\mathfrak{t}_{2}^{\varsigma-1}-\mathfrak{t}_{1}^{\varsigma-1}\right|}{\left(1+\mathfrak{t}_{2}^{\zeta-1}\right)\left(1+\mathfrak{t}_{1}^{\mathfrak{s}^{-1}}\right)} \frac{\mathfrak{p}(\mu)}{\Lambda}\left(\frac{\sum_{i=1}^{m} \mathrm{a}_{i 1} \boldsymbol{\eta}_{i}^{\varsigma-1}}{\Gamma_{\mathrm{q}}(\varsigma)}\right. \\
& \left.+\frac{\sum_{i=12}^{m} \mathrm{a}_{i 2} \eta_{i}^{\zeta-\nu-1}}{\Gamma_{\mathrm{q}}(\varsigma-v)}\right) \int_{0}^{\infty} \psi(\xi) \mathrm{d}_{\mathrm{q}} \xi \rightarrow 0 .
\end{aligned}
$$

Hence, $\mathfrak{H}(\mathbb{Q})$ is equicontinuous on any compact intervals of $J$.

STEP C: Let us prove that $\mathfrak{H}$ is equiconvergent at $\infty$. For any $\wp \in \mathbb{Q}$, by (A5)

$$
\int_{0}^{\infty} \mathfrak{w}(\xi, \wp(\xi)) \mathrm{d}_{\mathrm{q}} \xi \leq \mathfrak{p}(\mu) \int_{0}^{\infty} \psi(\xi) \mathrm{d}_{\mathrm{q}} \xi<\infty
$$

we have

$$
\begin{aligned}
& \lim _{\mathfrak{t} \rightarrow \infty}\left|\frac{\mathfrak{H} \wp}{1+\mathfrak{t}^{-1}}\right|=\lim _{\mathfrak{t} \rightarrow \infty} \mid \int_{0}^{\infty} \frac{{ }^{\mathbb{G}_{\mathrm{q}}(\mathfrak{t}, \xi)}}{1+\mathfrak{t}^{-1}} \mathfrak{w}(\xi, \wp(\xi)) \mathrm{d}_{\mathrm{q}} \xi \\
& +\frac{\mathfrak{t}^{5-1}}{\Lambda} \sum_{i=1}^{m}{ }_{1} \mathrm{a}_{i} \int_{0}^{\infty} \frac{{ }_{2} \mathbb{G}_{\mathrm{q}}\left({ }_{1} \eta_{i}, \xi\right)}{1+\mathfrak{t}^{-1}} \mathfrak{w}(\xi, \wp(\xi)) \mathrm{d}_{\mathrm{q}} \xi \\
& +\frac{\mathfrak{t}^{\zeta-1}}{\Lambda} \sum_{i=1}^{m}{ }_{2} \mathrm{a}_{i} \int_{0}^{\infty} \frac{{ }_{2} \mathbb{G}_{\mathrm{q}}\left({ }_{2} \eta_{i}, \xi\right)}{1+\mathfrak{t}^{5-1}} \mathfrak{w}(\xi, \wp(\xi)) \mathrm{d}_{\mathrm{q}} \xi \\
& \leq\left(\frac{1}{\Gamma_{\mathrm{q}}(\varsigma)}+\frac{\sum_{i=1}^{m} \mathrm{a}_{i 1} \eta_{i}^{\varsigma-1}}{\Lambda \Gamma_{\mathrm{q}}(\varsigma)}\right. \\
& \left.+\frac{\sum_{i=12}^{m} \mathrm{a}_{i 2} \eta_{i}^{\zeta-v-1}}{\Lambda \Gamma_{\mathrm{q}}(\varsigma-v)}\right) \int_{0}^{\infty}\left|\mathfrak{w}\left(\xi, \frac{\wp(\xi)\left(1+\xi^{\varsigma^{-1}}\right)}{1+\xi^{\varsigma^{-1}}}\right)\right| \mathrm{d}_{\mathrm{q}} \xi \\
& \leq\left(\frac{1}{\Gamma_{\mathrm{q}}(\varsigma)}+\frac{\sum_{i=1}^{m} \mathrm{a}_{i 1} \eta_{i}^{\varsigma-1}}{\Lambda \Gamma_{\mathrm{q}}(\varsigma)}\right. \\
& \left.+\frac{\sum_{i=12}^{m} \mathrm{a}_{i 2} \eta_{i}^{\varsigma-v-1}}{\Lambda \Gamma_{\mathrm{q}}(\varsigma-v)}\right) \int_{0}^{\infty}\left|\mathfrak{w}_{*}\left(\xi, \frac{\wp(\xi)}{1+\xi^{\varsigma-1}}\right)\right| \mathrm{d}_{\mathrm{q}} \xi \\
& \leq \mathfrak{p}(\mu) \nabla \int_{0}^{\infty} \psi(\xi) \mathrm{d}_{\mathrm{q}} \xi<\infty .
\end{aligned}
$$


Thus, we obtain

$$
\lim _{\mathfrak{t} \rightarrow+\infty}\left|\frac{\mathfrak{H} \wp(\mathfrak{t})}{1+\mathfrak{t}^{-1}}\right| \rightarrow 0
$$

Hence, $\mathfrak{H}: \mathbb{P} \rightarrow \mathbb{P}$ is equiconvergent at infinity. Thus, the operator $\mathfrak{H}$ is completely continuous. The proof is completed.

In the next subsections, the boundary value problem's (4) existence and uniqueness are shown via the Banach fixed-point theorem, the Schauder fixed-point theorem, and the Leray-Schauder nonlinear alternative theorem.

\subsection{Existence via the Leray-Schauder nonlinear alternative theorem}

Theorem 3.3 Suppose that $\exists \mu>0$, and functions $\mathfrak{p}$ and $\psi$ satisfy:

$$
\mu>\nabla \mathfrak{p}(\mu) \int_{0}^{\infty} \psi(\xi) \mathrm{d}_{\mathrm{q}} \xi
$$

and conditions (A5) and (A6) hold. Then, the boundary value problem (4) has an unbounded solution $\wp(\mathfrak{t})$ such that

$$
0 \leq \frac{\wp(\mathfrak{t})}{1+\mathfrak{t}^{5-1}} \leq \mu \quad(\mathfrak{t} \in J) .
$$

Proof Let

$$
\Upsilon=\{\wp \in \mathbb{P}:\|\wp\| \leq \mu\},
$$

we have $\wp \neq \lambda \mathfrak{H} \wp$ for $\wp \in \partial \Upsilon$ and $\lambda \in(0,1)$. If $\exists \wp \in \partial \Upsilon$ with $\wp=\lambda \mathfrak{H} \wp$, then for $\lambda \in(0,1)$, we obtain

$$
\begin{aligned}
\|\wp\|= & \sup _{\mathfrak{t} \in J}\left|\frac{\lambda \mathfrak{H} \wp(\mathfrak{t})}{1+\mathfrak{t}^{5-1}}\right| \leq \sup _{\mathfrak{t} \in J}\left|\frac{\mathfrak{t} \wp(\mathfrak{t})}{1+\mathfrak{t}^{5-1}}\right| \\
\leq & \sup _{\mathfrak{t} \in J}\left(\int_{0}^{\infty} \frac{\mathbb{G}_{\mathrm{q}}(\mathfrak{t}, \xi)}{1+\mathfrak{t}^{5-1}} \mathfrak{w}(\xi, \wp(\xi)) \mathrm{d}_{\mathrm{q}} \xi\right. \\
& +\frac{\mathfrak{t}^{\zeta-1}}{\Lambda} \sum_{i=1}^{m}{ }_{1} \mathrm{a}_{i} \int_{0}^{\infty} \frac{{ }_{2} \mathbb{G}_{\mathrm{q}}\left({ }_{1} \eta_{i}, \xi\right)}{1+\mathfrak{t}^{5}-1} \mathfrak{w}(\xi, \wp(\xi)) \mathrm{d}_{\mathrm{q}} \xi \\
& \left.+\frac{\mathfrak{t}^{\zeta-1}}{\Lambda} \sum_{i=1}^{m}{ }_{2} \mathrm{a}_{i} \int_{0}^{\infty} \frac{{ }_{2} \mathbb{G}_{\mathrm{q}}\left({ }_{2} \eta_{i}, \xi\right)}{1+\mathfrak{t}^{5-1}} \mathfrak{w}(\xi, \wp(\xi)) \mathrm{d}_{\mathrm{q}} \xi\right) \\
\leq & \frac{1}{\Gamma_{\mathrm{q}}(\varsigma)} \int_{0}^{\infty}|\mathfrak{w}(\xi, \wp(\xi))| \mathrm{d}_{\mathrm{q}} \xi \\
& +\frac{\sum_{i=1}^{m} \mathrm{a}_{i 1} \eta_{i}^{\varsigma-1}}{\Lambda \Gamma_{\mathrm{q}}(\varsigma)} \int_{0}^{\infty}|\mathfrak{w}(\xi, \wp(\xi))| \mathrm{d}_{\mathrm{q}} \xi \\
& +\frac{\sum_{i=1}^{m} \mathrm{a}_{i 2} \eta_{i}^{\zeta-\nu-1}}{\Lambda \Gamma_{\mathrm{q}}(\varsigma-v)} \int_{0}^{\infty} \mathfrak{w}(\xi, \wp(\xi)) \mathrm{d}_{\mathrm{q}} \xi \\
= & \left(\frac{1}{\Gamma_{\mathrm{q}}(\varsigma)}+\frac{\sum_{i=1}^{m} \mathrm{a}_{i 1} \eta_{i}^{\varsigma-1}}{\Lambda \Gamma_{\mathrm{q}}(\varsigma)}\right.
\end{aligned}
$$




$$
\begin{aligned}
& \left.+\frac{\sum_{i=1}^{m} \mathrm{a}_{i 2} \eta_{i}^{\varsigma-\nu-1}}{\Lambda \Gamma_{\mathrm{q}}(\varsigma-v)}\right) \int_{0}^{\infty}\left|\mathfrak{w}\left(\xi, \frac{\wp(\xi)\left(1+\xi^{\varsigma-1}\right)}{1+\xi^{-1}}\right)\right| \mathrm{d}_{\mathrm{q}} \xi \\
= & \left(\frac{1}{\Gamma_{\mathrm{q}}(\varsigma)}+\frac{\sum_{i=1}^{m} \mathrm{a}_{i 1} \eta_{i}^{\varsigma-1}}{\Lambda \Gamma_{\mathrm{q}}(\varsigma)}\right. \\
& \left.+\frac{\sum_{i=11}^{m} \eta_{i 2} \eta_{i}^{\zeta-\nu-1}}{\Lambda \Gamma_{\mathrm{q}}(\varsigma-\nu)}\right) \int_{0}^{\infty}\left|\mathfrak{w}_{*}\left(\xi, \frac{\wp(\xi)}{1+\xi^{-1}}\right)\right| \mathrm{d}_{\mathrm{q}} \xi \\
\leq & \mathfrak{p}(\mu) \nabla \int_{0}^{\infty} \psi(\xi) \mathrm{d}_{\mathrm{q}} \xi<\infty .
\end{aligned}
$$

Hence, for $\wp \in \partial \Upsilon$, we find

$$
\mu \leq \mathfrak{p}(\mu) \nabla \int_{0}^{\infty} \psi(\xi) \mathrm{d}_{\mathrm{q}} \xi
$$

which contradicts (18). By the fixed-point theorem of Schauder, the problem (4) has an unbounded solution $\wp=\wp(\mathfrak{t})$,

$$
0 \leq \wp(\mathfrak{t}) \leq \mu\left(1+\mathfrak{t}^{\varsigma^{-1}}\right), \quad \forall \mathfrak{t} \in J
$$

The proof is completed.

\subsection{Existence via the Schauder FpThm}

Theorem 3.4 From (A4), the boundary value problem (4) has at least 1 solution on $\mathfrak{t} \in J$.

Proof Assume that an operator $\mathfrak{H}$ is defined in (14). By testing to see that all Schauder fixed-point theorem's axioms on $\mathcal{C}_{\infty}$ are satisfied. $\mathfrak{w}$ and ${ }_{1} \mathbb{G}_{\mathrm{q}},{ }_{2} \mathbb{G}_{\mathrm{q}}$ are given continuous functions indicating that the operator $\mathfrak{H}$ is continuous. The remainder of the Theorem 3.4 proof is divided as follows:

STEP A: Let

$$
\Upsilon=\left\{\wp \in \mathcal{C}_{\infty}:\|\wp\| \leq \mu\right\}
$$

closed ball. Then, we prove that $\mathfrak{H}: \Upsilon \rightarrow \Upsilon$. We select

$$
\mu \geq \mathfrak{p}(\mu) \nabla \int_{0}^{\infty} \psi(\xi) \mathrm{d}_{\mathrm{q}} \xi
$$

For any $\wp \in \mathcal{C}_{\infty}$, we prove that $\mathfrak{H} \Upsilon \subset \Upsilon$. Hence, for $\mathfrak{t} \in J$, we obtain:

$$
\begin{aligned}
\left|\frac{\mathfrak{H} \wp(\mathfrak{t})}{1+\mathfrak{t}^{-1}}\right| \leq & \sup _{\mathfrak{t} \in J}\left(\int_{0}^{\infty} \frac{{ }_{0} \mathbb{G}_{\mathrm{q}}(\mathfrak{t}, \xi)}{1+\mathfrak{t}^{5-1}} \mathfrak{w}(\xi, \wp(\xi)) \mathrm{d}_{\mathrm{q}} \xi\right. \\
& +\frac{\mathfrak{t}^{\varsigma-1}}{\Lambda} \sum_{i=1}^{m}{ }_{1} \mathrm{a}_{i} \int_{0}^{\infty} \frac{{ }_{1} \mathbb{G}_{\mathrm{q}}\left({ }_{1} \eta_{i}, \xi\right)}{1+\mathfrak{t}^{5-1}} \mathfrak{w}(\xi, \wp(\xi)) \mathrm{d}_{\mathrm{q}} \xi \\
& \left.+\frac{\mathfrak{t}^{\varsigma-1}}{\Lambda} \sum_{i=1}^{m}{ }_{2} \mathrm{a}_{i} \int_{0}^{\infty} \frac{{ }_{2} \mathbb{G}_{\mathrm{q}}\left({ }_{2} \eta_{i}, \xi\right)}{1+\mathfrak{t}^{-1}} \mathfrak{w}(\xi, \wp(\xi)) \mathrm{d}_{\mathrm{q}} \xi\right) \\
\leq & \frac{1}{\Gamma_{\mathrm{q}}(\varsigma)} \int_{0}^{\infty}|\mathfrak{w}(\xi, \wp(\xi))| \mathrm{d}_{\mathrm{q}} \xi
\end{aligned}
$$




$$
\begin{aligned}
& +\frac{\sum_{i=1}^{m}{ }_{1} \mathrm{a}_{i 1} \eta_{i}^{\zeta-1}}{\Lambda \Gamma_{\mathrm{q}}(\varsigma)} \int_{0}^{\infty}|\mathfrak{w}(\xi, \wp(\xi))| \mathrm{d}_{\mathrm{q}} \xi \\
& +\frac{\sum_{i=12}^{m} \mathrm{a}_{i 2} \eta_{i}^{\zeta-\nu-1}}{\Lambda \Gamma_{\mathrm{q}}(\varsigma-v)} \int_{0}^{\infty} \mathfrak{w}(\xi, \wp(\xi)) \mathrm{d}_{\mathrm{q}} \xi \\
= & \left(\frac{1}{\Gamma_{\mathrm{q}}(\varsigma)}+\frac{\sum_{i=11}^{m} \mathrm{a}_{i 1} \eta_{i}^{\zeta-1}}{\Lambda \Gamma_{\mathrm{q}}(\varsigma)}\right. \\
& \left.+\frac{\sum_{i=1}^{m}{ }_{2} \mathrm{a}_{i 2} \eta_{i}^{\zeta-v-1}}{\Lambda \Gamma_{\mathrm{q}}(\varsigma-v)}\right) \int_{0}^{\infty}\left|\mathfrak{w}\left(\xi, \frac{\wp(\xi)\left(1+\xi^{5-1}\right)}{1+\xi^{5-1}}\right)\right| \mathrm{d}_{\mathrm{q}} \xi \\
= & \left(\frac{1}{\Gamma_{\mathrm{q}}(\varsigma)}+\frac{\sum_{i=1}^{m} \mathrm{a}_{i 1} \eta_{i}^{\zeta-1}}{\Lambda \Gamma_{\mathrm{q}}(\varsigma)}\right. \\
& \left.+\frac{\sum_{i=1}^{m} \mathrm{a}_{i 2} \eta_{i}^{\zeta-v-1}}{\Lambda \Gamma_{\mathrm{q}}(\varsigma-v)}\right) \int_{0}^{\infty}\left|\mathfrak{w} *\left(\xi, \frac{\wp(\xi)}{1+\xi \xi^{-1}}\right)\right| \mathrm{d}_{\mathrm{q}} \xi \\
\leq & \mathfrak{p}(\mu) \nabla \int_{0}^{\infty} \psi(\xi) \mathrm{d}_{\mathrm{q}} \xi<\infty .
\end{aligned}
$$

As a result, $\|\mathfrak{H} \wp\|_{\mathcal{C}_{\infty}} \leq \mu$, which indicates that $\mathfrak{H} \Upsilon \subset \Upsilon$, i.e., the operator $\mathfrak{H}$ maps $\Upsilon$ into $\Upsilon$.

STEP B: From Lemma (3.2), we need to prove that $\mathfrak{H}$ is continuous and completely continuous on $\mathcal{C}_{\infty}$. Thus, by the Schauder fixed-point theorem, the operator $\mathfrak{H}$ has a fixed point $\wp$ in $\mathcal{C}_{\infty}$ which is a solution of problem (4).

\subsection{Existence and uniqueness results via the Banach FpThm}

Theorem 3.5 Let (A5) and a hypothesis hold (A6), $\exists$ is a positive function $\varrho(\mathfrak{t})$ with

$$
\varrho^{*}=\int_{0}^{\infty}\left(1+\xi^{\varsigma^{-1}}\right) \varrho(\xi) \mathrm{d} \xi<\infty
$$

and

$$
|\mathfrak{w}(\mathfrak{t}, \wp)-\mathfrak{w}(\mathfrak{t}, \wp)| \leq \varrho(\mathfrak{t})|\wp-\wp|, \quad \forall, \mathfrak{t} \in J, \wp, \wp \in \mathbb{R}
$$

Then, the boundary value problem (4) has a unique solution: $\wp(\mathfrak{t})$ in $\mathcal{C}_{\infty}$. Moreover, $\exists$ is a monotone iterative sequence $\left\{\wp_{n}(\mathfrak{t})\right\}$ such that $\wp_{n}(\mathfrak{t}) \rightarrow \wp(\mathfrak{t})$ as $n \rightarrow \infty$, which is uniform on any unbounded subdomain of $\mathfrak{t} \in \wp$, where

$$
\wp_{n}(\mathfrak{t})=\int_{0}^{\infty}{ }_{1} \mathbb{G}_{\mathrm{q}}(\mathfrak{t}, \xi) \mathfrak{w}\left(\xi, \wp_{n-1}(\xi)\right) \mathrm{d} \xi
$$

Furthermore, $\exists$ is an error estimate for the following:

$$
\left\|\wp_{n}-\wp\right\|_{\infty} \leq \frac{\theta^{n}}{1-\theta}\left\|\wp_{1}-\wp_{0}\right\|_{\infty} \quad(n=1,2, \ldots)
$$

and

$$
\theta=\left(\frac{1}{\Gamma_{\mathrm{q}}(\varsigma)}+\frac{\sum_{i=11}^{m} \mathrm{a}_{i 1} \eta_{i}^{\zeta-1}}{\Lambda \Gamma_{\mathrm{q}}(\varsigma)}+\frac{\sum_{i=12}^{m} \mathrm{a}_{i 2} \eta_{i}^{\zeta-\nu-1}}{\Lambda \Gamma_{\mathrm{q}}(\varsigma-v)}\right) \varrho^{*}<1
$$


Proof By considering an operator $\mathfrak{H}$ defined by the equation (14). Assume that $\wp_{1}, \wp_{2} \in$ $\mathcal{C}_{\infty}$. For $\mathfrak{t} \in J$, we have

$$
\begin{aligned}
& \left|\frac{1}{1+\mathfrak{t}^{5-1}}\left(\mathfrak{H} \wp_{1}(\mathfrak{t})-\mathfrak{H} \wp_{2}(\mathfrak{t})\right)\right| \\
& \leq \sup _{\mathfrak{t} \in J}\left(\int_{0}^{\infty} \frac{{ }_{1} \mathbb{G}_{\mathbf{q}}(\mathfrak{t}, \xi)}{1+\mathfrak{t}^{5}-1}\left|\mathfrak{w}\left(\xi, \wp_{1}(\xi)\right)-\mathfrak{w}\left(\xi, \wp_{2}(\xi)\right)\right| \mathrm{d}_{\mathrm{q}} \xi\right. \\
& +\frac{t^{\varsigma^{-1}}}{\Lambda} \sum_{i=1}^{m}{ }_{1} \mathrm{a}_{i} \int_{0}^{\infty} \frac{{ }_{2} \mathbb{G}_{\mathrm{q}}\left({ }_{1} \eta_{i}, \xi\right)}{1+\mathfrak{t}^{-1}}\left|\mathfrak{w}\left(\xi, \wp_{1}(\xi)\right)-\mathfrak{w}\left(\xi, \wp_{2}(\xi)\right)\right| \mathrm{d}_{\mathrm{q}} \xi \\
& \left.+\frac{\mathfrak{t}^{5-1}}{\Lambda} \sum_{i=1}^{m}{ }_{2} \mathrm{a}_{i} \int_{0}^{\infty} \frac{{ }_{2} \mathbb{G}_{\mathrm{q}}\left({ }_{2} \eta_{i}, \xi\right)}{1+\mathfrak{t}^{5-1}}\left|\mathfrak{w}\left(\xi, \wp_{1}(\xi)\right)-\mathfrak{w}\left(\xi, \wp_{2}(\xi)\right)\right| \mathrm{d}_{\mathrm{q}} \xi\right) \\
& \leq \frac{1}{\Gamma_{\mathrm{q}}(\varsigma)} \int_{0}^{\infty}\left|\mathfrak{w}\left(\xi, \wp_{1}(\xi)\right)-\mathfrak{w}\left(\xi, \wp_{2}(\xi)\right)\right| \mathrm{d}_{\mathrm{q}} \xi \\
& +\frac{\sum_{i=11}^{m} \mathrm{a}_{i 1} \eta_{i}^{\varsigma-1}}{\Lambda \Gamma_{\mathrm{q}}(\varsigma)} \int_{0}^{\infty}\left|\mathfrak{w}\left(\xi, \wp_{1}(\xi)\right)-\mathfrak{w}\left(\xi, \wp_{2}(\xi)\right)\right| \mathrm{d}_{\mathrm{q}} \xi \\
& +\frac{\sum_{i=1}^{m} 2 \mathrm{a}_{i 2} \eta_{i}^{\varsigma-v-1}}{\Lambda \Gamma_{\mathrm{q}}(\varsigma-v)} \int_{0}^{\infty}\left|\mathfrak{w}\left(\xi, \wp_{1}(\xi)\right)-\mathfrak{w}\left(\xi, \wp_{2}(\xi)\right)\right| \mathrm{d}_{\mathrm{q}} \xi \\
& \leq\left(\frac{1}{\Gamma_{\mathrm{q}}(\varsigma)}+\frac{\sum_{i=1}^{m} \mathrm{a}_{i 1} \eta_{i}^{\varsigma-1}}{\Lambda \Gamma_{\mathrm{q}}(\varsigma)}+\frac{\sum_{i=12}^{m} \mathrm{a}_{i 2} \eta_{i}^{\varsigma-\nu-1}}{\Lambda \Gamma_{\mathrm{q}}(\varsigma-v)}\right) \\
& \times \int_{0}^{\infty} \mid \mathfrak{w}\left(\xi, \frac{\wp_{1}(\xi)\left(1+\xi^{\varsigma-1}\right)}{1+\xi^{\varsigma^{-1}}}\right) \\
& -\mathfrak{w}\left(\xi, \frac{\wp_{2}(\xi)\left(1+\xi^{\varsigma-1}\right)}{1+\xi^{\varsigma^{-1}}}\right) \mid \mathrm{d}_{\mathrm{q}} \xi \\
& \leq\left(\frac{1}{\Gamma_{\mathrm{q}}(\varsigma)}+\frac{\sum_{i=1}^{m} \mathrm{a}_{i 1} \eta_{i}^{\varsigma-1}}{\Lambda \Gamma_{\mathrm{q}}(\varsigma)}+\frac{\sum_{i=12}^{m} \mathrm{a}_{i 2} \eta_{i}^{\varsigma-\nu-1}}{\Lambda \Gamma_{\mathrm{q}}(\varsigma-v)}\right) \\
& \times \int_{0}^{\infty}\left|\mathfrak{w}_{*}\left(\xi, \frac{\wp_{1}(\xi)}{1+\xi^{\varsigma^{-1}}}\right)-\mathfrak{w}_{*}\left(\xi, \frac{\wp_{2}(\xi)}{1+\xi^{\varsigma^{-1}}}\right)\right| \mathrm{d}_{\mathrm{q}} \xi \\
& \leq\left(\frac{1}{\Gamma_{\mathrm{q}}(\varsigma)}+\frac{\sum_{i=1}^{m}{ }_{1} \mathrm{a}_{i 1} \eta_{i}^{\varsigma-1}}{\Lambda \Gamma_{\mathrm{q}}(\varsigma)}+\frac{\sum_{i=12}^{m} \mathrm{a}_{i 2} \eta_{i}^{\varsigma-\nu-1}}{\Lambda \Gamma_{\mathrm{q}}(\varsigma-v)}\right) \\
& \times\left(\int_{0}^{\infty}\left[\left(1+\xi^{\varsigma-1}\right) \varrho(\xi)\right] \mathrm{d} \xi\right)\left\|\wp_{1}-\wp_{2}\right\|_{\infty} .
\end{aligned}
$$

Consequently,

$$
\begin{aligned}
& \left|\left(\mathfrak{H} \wp_{1}\right)(\mathfrak{t})-\left(\mathfrak{H} \wp_{2}\right)(\mathfrak{t})\right| \\
& \quad \leq\left(\frac{1}{\Gamma_{\mathrm{q}}(\varsigma)}+\frac{\sum_{i=1}^{m} \mathrm{a}_{i 1} \eta_{i}^{\zeta-1}}{\Lambda \Gamma_{\mathrm{q}}(\varsigma)}+\frac{\sum_{i=1}^{m} \mathrm{a}_{i 2} \eta_{i}^{\zeta-\nu-1}}{\Lambda \Gamma_{\mathrm{q}}(\varsigma-v)}\right) \varrho^{*}\|\wp-\wp\|_{\infty} \\
& \quad=\theta\left\|\wp_{1}-\wp_{2}\right\|_{\infty} .
\end{aligned}
$$

Thus, we collect that

$$
\left\|\mathfrak{H} \wp_{1}-\mathfrak{H} \wp_{2}\right\|_{\infty} \leq \theta\left\|\wp_{1}-\wp_{2}\right\|_{\infty}, \quad \forall \wp_{1}, \wp_{2} \in \mathcal{C}_{\infty} .
$$


As $\theta<1$, then the Banach fixed-point theorem ensures that $\mathfrak{H}$ has a unique fixed point $\wp$ in $\mathcal{C}_{\infty}$. Hence, the problem (4) has a unique solution $\wp \in \mathcal{C}_{\infty}$. Furthermore, for each $\wp_{0} \in \mathcal{C}_{\infty},\left\|\wp_{n}-\wp\right\|_{\infty} \rightarrow 0$ as $n \rightarrow \infty$, where $\wp_{n}=\mathfrak{H} \wp_{n-1}(n=1,2 \ldots)$. From (21), we find

$$
\left\|\wp_{n}-\wp_{n-1}\right\|_{\infty} \leq \theta^{n-1}\left\|\wp_{1}-\wp_{0}\right\|_{\infty}
$$

and

$$
\begin{aligned}
\left\|\wp_{n}-\wp_{j}\right\|_{\infty} \leq & \left\|\wp_{n}-\wp_{n-1}\right\|_{\infty}+\left\|\wp_{n-1}-\wp_{n-2}\right\|_{\infty} \\
& +\cdots+\left\|\wp_{j+1}-\wp_{j}\right\|_{\infty} \\
= & \frac{\theta^{n}\left(1-\theta^{n-j}\right)}{1-\theta}\left\|\wp_{1}-\wp_{0}\right\|_{\infty} .
\end{aligned}
$$

By supposing that $n \rightarrow \infty$ on both sides of (22), we can estimate

$$
\left\|\wp_{n}-\wp\right\|_{\infty} \leq \frac{\theta^{n}}{1-\theta}\left\|\wp_{1}-\wp_{0}\right\|_{\infty}
$$

Hence equation (19) holds, and this theorem's proof is completed.

\section{Applications via numerical results}

Example 4.1 By taking

$$
\varsigma=\frac{7}{2} \in(3,4], \quad v=\frac{9}{5}>0,
$$

and $q=\left\{\frac{1}{8}, \frac{1}{2}, \frac{10}{13}\right\}$, let us have the following boundary value problem for nonlinear fractional q-difference equations on an unbounded domain:

$$
\left\{\begin{array}{l}
\mathbb{D}_{\mathrm{q}}^{\frac{7}{2}} \wp(\mathfrak{t})=\frac{\ln (1+\mathfrak{t})}{\left(1+\mathfrak{t}^{2}\right)^{2}}+\frac{e^{-3 t}}{1+\mathfrak{t}^{2}} \sin \left(3 \mathfrak{t}^{2}+|\wp(\mathfrak{t})|\right), \quad \mathfrak{t} \in J, \\
\wp(0)=\wp^{\prime}(0)=\wp^{\prime \prime}(0)=0, \\
\mathbb{D}_{\mathrm{q}}^{\frac{5}{2}} \wp(\infty)=\sum_{i=1}^{m} \mathrm{a}_{i} \wp\left({ }_{1} \eta_{i}\right)+\sum_{i=1}^{m}{ }_{2} \mathrm{a}_{i} \mathbb{D}_{\mathrm{q}}^{2} \wp\left({ }_{2} \eta_{i}\right),
\end{array}\right.
$$

where

$$
\mathfrak{w}(\mathfrak{t}, \wp)=\frac{\ln (1+\mathfrak{t})}{\left(1+\mathfrak{t}^{2}\right)^{2}}+\frac{e^{-3 \mathfrak{t}}}{\left(1+\mathfrak{t}^{2}\right)} \sin \left(3 \mathfrak{t}^{2}+|\wp(\mathfrak{t})|\right),
$$

and ${ }_{1} \mathrm{a}_{i},{ }_{2} \mathrm{a}_{i},{ }_{1} \eta_{i, 2} \eta_{i}$ satisfy

$$
\begin{aligned}
0 & \leq{ }_{1} \mathrm{a}_{i 1} \eta_{i}^{\frac{5}{2}}+{ }_{2} \mathrm{a}_{i 2} \eta_{i}^{\frac{1}{2}} \\
& =1 \times \frac{1}{4}+1 \times \frac{2}{3}=0.9166<\Gamma_{\mathrm{q}}(\varsigma)= \begin{cases}1.2076, & \mathrm{q}=\frac{1}{8} \\
2.3814, & \mathrm{q}=\frac{1}{2} \\
5.5035, & \mathrm{q}=\frac{10}{13}\end{cases}
\end{aligned}
$$

whenever, we take

$$
{ }_{1} \eta_{i}=\frac{1}{4}, \quad{ }_{2} \eta_{i}=\frac{2}{3}, \quad{ }_{1} \mathrm{a}_{i}={ }_{2} \mathrm{a}_{i}=1 .
$$


Table 1 Numerical results of $\Lambda, \varrho^{*}$ and $\theta$ for $\mathrm{q}=\frac{1}{8}, \frac{1}{2}$ in Example 4.1

\begin{tabular}{rlllllll}
\hline$n$ & $\Gamma_{\mathrm{q}}(\varsigma)$ & $\Gamma_{\mathrm{q}}(v)$ & $\Gamma_{\mathrm{q}}(\zeta-v)$ & $\Lambda$ & $\theta_{0}$ & $\varrho^{*}$ & $\theta$ \\
\hline & $\mathrm{q}=\frac{1}{8}$ & & & & & & \\
1 & 1.2102 & 1.2112 & 1.2705 & 0.4618 & 2.1656 & 0.2192 & 0.4747 \\
2 & 1.2079 & 1.2124 & 1.2726 & 0.4621 & 2.1642 & 0.2192 & 0.4744 \\
3 & 1.2077 & 1.2125 & 1.2729 & 0.4621 & 2.1641 & 0.2192 & 0.4744 \\
4 & 1.2076 & 1.2125 & 1.2729 & 0.4621 & 2.1640 & 0.2192 & 0.4744 \\
5 & 1.2076 & 1.2126 & 1.2729 & 0.4621 & 2.1640 & 0.2192 & 0.4744 \\
& $\mathrm{q}=\frac{1}{2}$ & & & & & & \\
1 & 2.8267 & 2.1519 & 2.2893 & 1.8658 & 0.5360 & 0.2192 & 0.1175 \\
2 & 2.5877 & 2.1986 & 2.3674 & 1.7335 & 0.5769 & 0.2192 & 0.1265 \\
3 & 2.4808 & 2.2206 & 2.4045 & 1.6728 & 0.5978 & 0.2192 & 0.1311 \\
4 & 2.4301 & 2.2313 & 2.4226 & 1.6436 & 0.6084 & 0.2192 & 0.1334 \\
5 & 2.4054 & 2.2366 & 2.4315 & 1.6294 & 0.6137 & 0.2192 & 0.1345 \\
6 & 2.3933 & 2.2392 & 2.4359 & 1.6223 & 0.6164 & 0.2192 & 0.1351 \\
7 & 2.3872 & 2.2405 & 2.4381 & 1.6188 & 0.6177 & 0.2192 & 0.1354 \\
8 & 2.3842 & 2.2412 & 2.4392 & 1.6170 & 0.6184 & 0.2192 & 0.1356 \\
9 & 2.3827 & 2.2415 & 2.4398 & 1.6162 & 0.6188 & 0.2192 & 0.1356 \\
10 & 2.3819 & 2.2417 & 2.4401 & 1.6157 & 0.6189 & 0.2192 & $\underline{0.1357}$ \\
11 & 2.3816 & 2.2417 & 2.4402 & 1.6155 & 0.6190 & 0.2192 & 0.1357 \\
12 & 2.3814 & 2.2418 & 2.4403 & 1.6154 & 0.6190 & 0.2192 & 0.1357 \\
\hline
\end{tabular}

We can see the results of $\Lambda$ and $\theta$ in Tables 1 and 2. These results are plotted in Fig. 1 . Then (A4) holds. Secondly, we obtain

$$
|\mathfrak{w}(\mathfrak{t}, \wp)-\mathfrak{w}(\mathfrak{t}, \wp)|=\frac{e^{-3 \mathfrak{t}}|\wp-\wp|}{5\left(1+\mathfrak{t}^{2 \cdot 5}\right)}
$$

By taking $\varrho(\mathfrak{t})=\frac{e^{-\mathfrak{t}}}{5\left(1+\mathfrak{t}^{2}\right)}$, we directly obtain:

$$
\varrho^{*}=\int_{0}^{+\infty} \varrho(\xi)\left(1+\xi^{\varsigma^{-1}}\right) \mathrm{d} \xi=\frac{1}{5}<+\infty .
$$

We shall check that condition (20) is satisfied. By using the MATLAB program Additional file 1, Algorithm 1) according to Eq. (14), we find

$$
\theta=\theta_{0} \varrho^{*} \simeq\left\{\begin{array}{ll}
0.4744, & \mathrm{q}=\frac{1}{8} \\
0.1357, & \mathrm{q}=\frac{1}{2}, \\
0.0465, & \mathrm{q}=\frac{10}{13},
\end{array}\right\}<1
$$

where

$$
\theta_{0}=\frac{1}{\Gamma_{\mathrm{q}}(\varsigma)}+\frac{\sum_{i=1}^{m} \mathrm{a}_{i 1} \eta_{i}^{\varsigma-1}}{\Lambda \Gamma_{\mathrm{q}}(\varsigma)}+\frac{\sum_{i=1}^{m}{ }_{2} \mathrm{a}_{i 2} \eta_{i}^{\zeta-v-1}}{\Lambda \Gamma_{\mathrm{q}}(\varsigma-v)}
$$

Tables 1 and 2 show the results. Also, We can see a graphical representation of $\theta$ in Fig. 1 . Thus, from Theorem 3.5, the boundary value problem (23) has a unique solution.

Example 4.2 By taking

$$
\varsigma=\frac{17}{5} \in(3,4], \quad v=\frac{25}{9}>0
$$


Table 2 Numerical results of $\Lambda, \varrho^{*}$ and $\theta$ for $\mathrm{q}=\frac{10}{13}$ in Example 4.1

\begin{tabular}{|c|c|c|c|c|c|c|c|}
\hline$n$ & $\Gamma_{\mathrm{q}}(\varsigma)$ & $\Gamma_{\mathrm{q}}(v)$ & $\Gamma_{\mathrm{q}}(\varsigma-v)$ & $\Lambda$ & $\theta_{0}$ & $\varrho^{*}$ & $\theta$ \\
\hline & $q=\frac{10}{13}$ & & & & & & \\
\hline 1 & 12.7438 & 4.2729 & 4.3560 & 10.5099 & 0.0951 & 0.2192 & 0.0209 \\
\hline 2 & 10.0203 & 4.4743 & 4.6759 & 8.3756 & 0.1194 & 0.2192 & 0.0262 \\
\hline 3 & 8.5259 & 4.6081 & 4.8917 & 7.1824 & 0.1392 & 0.2192 & 0.0305 \\
\hline 4 & 7.6129 & 4.7014 & 5.0440 & 6.4453 & 0.1552 & 0.2192 & 0.0340 \\
\hline 5 & 7.0164 & 4.7686 & 5.1543 & 5.9602 & 0.1678 & 0.2192 & 0.0368 \\
\hline \multirow[t]{2}{*}{6} & 6.6088 & 4.8178 & 5.2355 & 5.6272 & 0.1777 & 0.2192 & 0.0390 \\
\hline & $\vdots$ & : & $\vdots$ & & $\vdots$ & & : \\
\hline 18 & 5.5407 & 4.9627 & 5.4771 & 4.7478 & 0.2106 & 0.2192 & 0.0462 \\
\hline 19 & 5.5312 & 4.9641 & 5.4794 & 4.7399 & 0.2110 & 0.2192 & 0.0463 \\
\hline 20 & 5.5239 & 4.9652 & 5.4813 & 4.7339 & 0.2112 & 0.2192 & 0.0463 \\
\hline 21 & 5.5183 & 4.9660 & 5.4827 & 4.7293 & 0.2115 & 0.2192 & 0.0464 \\
\hline 22 & 5.5140 & 4.9667 & 5.4838 & 4.7257 & 0.2116 & 0.2192 & 0.0464 \\
\hline 23 & 5.5107 & 4.9672 & 5.4846 & 4.7229 & 0.2117 & 0.2192 & 0.0464 \\
\hline 24 & 5.5081 & 4.9676 & 5.4852 & 4.7208 & 0.2118 & 0.2192 & 0.0464 \\
\hline 25 & 5.5062 & 4.9678 & 5.4857 & 4.7192 & 0.2119 & 0.2192 & $\underline{0.0465}$ \\
\hline 26 & 5.5047 & 4.9681 & 5.4861 & 4.7180 & 0.2120 & 0.2192 & 0.0465 \\
\hline 27 & 5.5035 & 4.9682 & 5.4864 & 4.7170 & 0.2120 & 0.2192 & 0.0465 \\
\hline
\end{tabular}

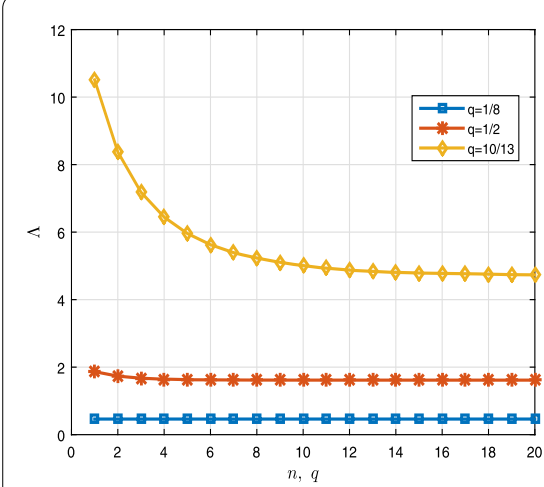

(a) $\Lambda$

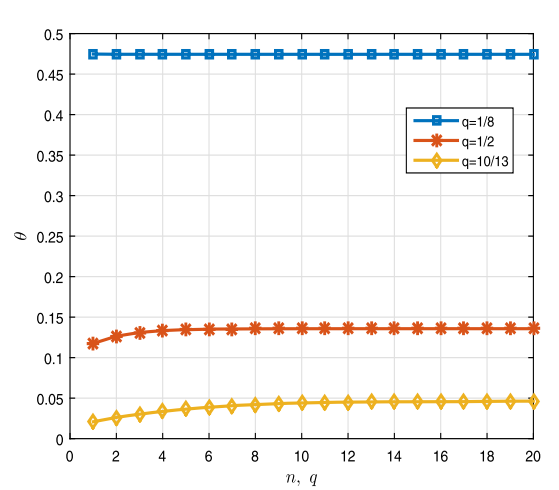

(b) $\theta$

Figure 1 Graphical representation of $\Lambda$ and $\theta$ for $\mathbf{q}=\frac{1}{8}, \frac{1}{2}, \frac{10}{13}$ in Example 4.1

and

$$
q=\left\{\frac{1}{7}, \frac{1}{2}, \frac{8}{9}\right\},
$$

let us have the following boundary value problem for nonlinear fractional q-difference equations on an unbounded domain:

$$
\left\{\begin{array}{l}
\mathbb{D}_{\mathrm{q}}^{\frac{17}{5}} \wp(\mathfrak{t})=\frac{e^{-2 \mathfrak{t}}|\wp(\mathfrak{t})|}{7\left(1+\sqrt[12]{\mathfrak{t}^{5}}\right)}, \quad \mathfrak{t} \in J, \\
\wp(0)=\wp^{\prime}(0)=\wp^{\prime \prime}(0)=0, \\
\mathbb{D}_{\mathrm{q}}^{\frac{12}{5}} \wp(\infty)=\sum_{i=1}^{m} \mathrm{a}_{i} \wp\left({ }_{1} \eta_{i}\right)+\sum_{i=1}^{m}{ }_{2} \mathrm{a}_{i} \mathbb{D}_{\mathrm{q}}^{\frac{25}{9}} \wp\left({ }_{2} \eta_{i}\right),
\end{array}\right.
$$

where

$$
\mathfrak{w}(\mathfrak{t}, \wp)=\frac{e^{-2 \mathfrak{t}}|\wp(\mathfrak{t})|}{7\left(1+\sqrt[12]{\mathfrak{t}^{5}}\right)},
$$


Table 3 Numerical results of $\Lambda, \nabla$ and $\mu$ for $\mathrm{q}=\frac{1}{7}$ in Example 4.2

\begin{tabular}{lllllll}
\hline$n$ & $\Gamma_{\mathrm{q}}(\varsigma)$ & $\Gamma_{\mathrm{q}}(v)$ & $\Gamma_{\mathrm{q}}(\varsigma-v)$ & $\Lambda$ & $\nabla$ & $\mu>\nabla \mathfrak{p}(\mu) \int_{0}^{\infty} \psi(\xi) \mathrm{d}_{\mathrm{q}} \xi$ \\
\hline & $\mathrm{q}=\frac{1}{7}$ and calculated $\mu=0.1$ & & & & \\
1 & 1.2287 & 1.1454 & -1.0393 & 2.7995 & 0.3572 & $0.1>0.0042$ \\
2 & 1.2253 & 1.1428 & -1.0823 & 2.6808 & 0.3730 & $0.1>0.0043$ \\
3 & 1.2249 & 1.1424 & -1.0885 & 2.6645 & 0.3753 & $\underline{0.1>0.0044}$ \\
4 & 1.2248 & 1.1423 & -1.0893 & 2.6622 & 0.3756 & $0.1>0.0044$ \\
5 & 1.2248 & 1.1423 & -1.0895 & 2.6619 & 0.3757 & $0.1>0.0044$ \\
\hline
\end{tabular}

Table 4 Numerical results of $\Lambda, \nabla$ and $\mu$ for $\mathrm{q}=\frac{1}{7}$ in Example 4.2

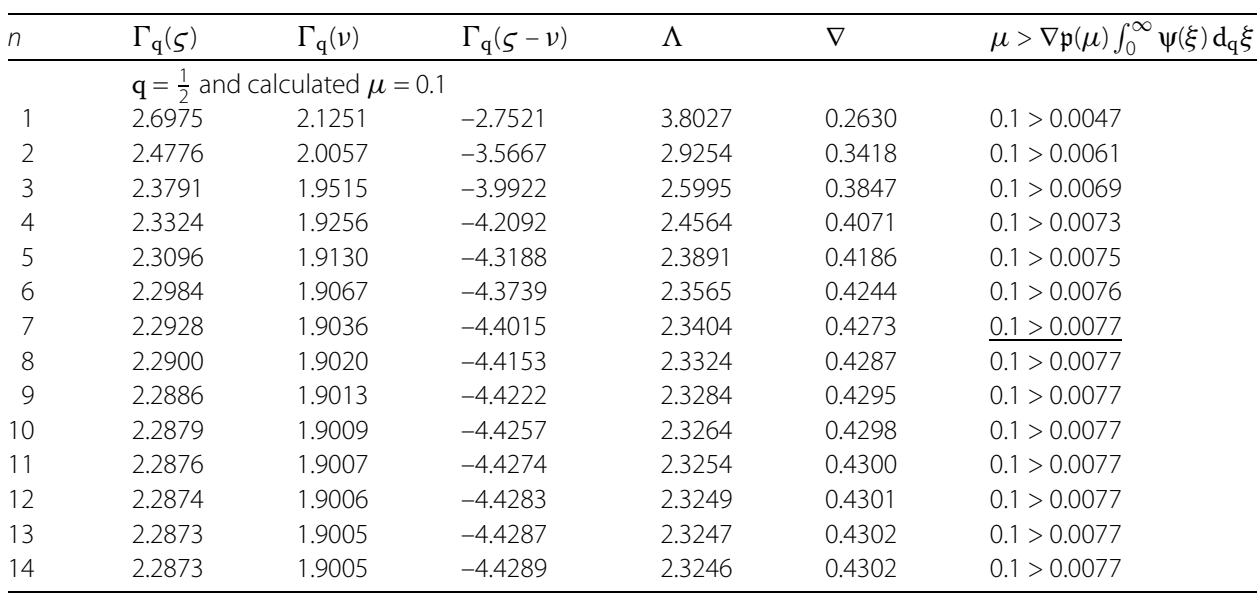

$$
\mathfrak{w}_{*}(\mathfrak{t}, \wp)=\mathfrak{w}\left(\mathfrak{t},\left(1+\mathfrak{t}^{\varsigma-1}\right) \wp\right)=\frac{e^{-2 \mathfrak{t}}\left|\left(1+\mathfrak{t}^{5^{-1}}\right) \wp(\mathfrak{t})\right|}{7\left(1+\sqrt[12]{\mathfrak{t}^{5}}\right)}=\frac{1}{7}|\wp(\mathfrak{t})| e^{-2 \mathfrak{t}}
$$

Choose $\mathfrak{p}(\wp)=|\wp(\mathfrak{t})|, \psi(\mathfrak{t})=e^{-2 \mathfrak{t}}$. For example, if we take

$$
\begin{array}{llll}
{ }_{1} \mathrm{a}_{1}=1, & { }_{1} \mathrm{a}_{2}=1.5, & { }_{2} \mathrm{a}_{1}=1, & { }_{2} \mathrm{a}_{2}=0.75, \\
{ }_{1} \eta_{1}=\frac{4}{5}, & { }_{1} \eta_{2}=\frac{2}{3}, & { }_{2} \eta_{1}=\frac{1}{3}, & { }_{2} \eta_{2}=\frac{7}{8},
\end{array}
$$

we have

$$
\left|\mathfrak{w}_{*}(\mathfrak{t}, \wp)-\mathfrak{w} *(\mathfrak{t}, \wp)\right|=\frac{1}{7} e^{-2 \mathfrak{t}}|\wp-\wp| \leq \frac{e^{-2 \mathfrak{t}}}{2}|\wp-\wp|,
$$

and

$$
\begin{aligned}
0 & \leq\left\{\sum_{i=1}^{m} a_{i} \xi_{i}^{\varsigma-1}+\sum_{i=1}^{m} b_{i} \xi_{i}^{\zeta-\nu-1} \frac{\Gamma_{\mathrm{q}}(\varsigma)}{\Gamma_{\mathrm{q}}(\varsigma-v)}\right\} \leq \Gamma_{\mathrm{q}}(\varsigma) \\
& = \begin{cases}-1.4396, & \mathrm{q}=\frac{1}{7}, \\
-0.0476, & \mathrm{q}=\frac{1}{2}, \\
-2.4611, & \mathrm{q}=\frac{8}{9} .\end{cases}
\end{aligned}
$$

Tables 3, 4 and 5 show the results. Also, We can see a graphical representation of

$$
\nabla \mathfrak{p}(\mu) \int_{0}^{\infty} \psi(\xi) \mathrm{d}_{\mathrm{q}} \xi
$$


Table 5 Numerical results of $\Lambda, \nabla$ and $\mu$ for $\mathrm{q}=\frac{8}{9}$ in Example 4.2

\begin{tabular}{|c|c|c|c|c|c|c|}
\hline$n$ & $\Gamma_{\mathrm{q}}(\varsigma)$ & $\Gamma_{\mathrm{q}}(\nu)$ & $\Gamma_{\mathrm{q}}(\varsigma-v)$ & $\Lambda$ & $\nabla$ & $\mu>\nabla \mathfrak{p}(\mu) \int_{0}^{\infty} \psi(\xi) \mathrm{d}_{\mathrm{q}} \xi$ \\
\hline & \multicolumn{6}{|c|}{$\mathrm{q}=\frac{8}{9}$ and calculated $\mu=0.1$} \\
\hline 1 & 55.9818 & 21.9886 & -3.1623 & 95.6034 & 0.0105 & $0.1>0.0000$ \\
\hline 2 & 41.2031 & 18.2245 & -5.4122 & 57.5855 & 0.0174 & $0.1>0.0000$ \\
\hline 3 & 32.8940 & 15.9102 & -7.6526 & 41.6420 & 0.0240 & $0.1>0.0000$ \\
\hline 4 & 27.6527 & 14.3446 & -9.8069 & 32.9949 & 0.0303 & $0.1>0.0000$ \\
\hline 5 & 24.0881 & 13.2182 & -11.8371 & 27.6229 & 0.0362 & $0.1>0.0000$ \\
\hline 6 & 21.5322 & 12.3725 & -13.7253 & 23.9933 & 0.0417 & $\underline{0.1>0.0001}$ \\
\hline 7 & 19.6267 & 11.7174 & -15.4655 & 21.3974 & 0.0467 & $0.1>0.0001$ \\
\hline \multirow[t]{2}{*}{8} & 18.1631 & 11.1978 & -17.0587 & 19.4632 & 0.0514 & $0.1>0.0001$ \\
\hline & $\vdots$ & $\vdots$ & & $\vdots$ & $\vdots$ & $\vdots$ \\
\hline 79 & 11.0024 & 8.3731 & -31.1770 & 10.6630 & 0.0938 & $0.1>0.0001$ \\
\hline 80 & 11.0023 & 8.3731 & -31.1774 & 10.6629 & 0.0938 & $0.1>0.0001$ \\
\hline 81 & 11.0022 & 8.3730 & -31.1777 & 10.6627 & 0.0938 & $0.1>0.0001$ \\
\hline 82 & 11.0021 & 8.3730 & -31.1780 & 10.6626 & 0.0938 & $0.1>0.0001$ \\
\hline 83 & 11.0020 & 8.3729 & -31.1783 & 10.6625 & 0.0938 & $0.1>0.0001$ \\
\hline 84 & 11.0019 & 8.3729 & -31.1786 & 10.6624 & 0.0938 & $0.1>0.0001$ \\
\hline 85 & 11.0019 & 8.3729 & -31.1788 & 10.6624 & 0.0938 & $0.1>0.0001$ \\
\hline 86 & 11.0018 & 8.3729 & -31.1790 & 10.6623 & 0.0938 & $0.1>0.0001$ \\
\hline 87 & 11.0017 & 8.3728 & -31.1792 & 10.6622 & 0.0938 & $0.1>0.0001$ \\
\hline 88 & 11.0017 & 8.3728 & -31.1793 & 10.6622 & 0.0938 & $0.1>0.0001$ \\
\hline 89 & 11.0016 & 8.3728 & -31.1794 & 10.6621 & 0.0938 & $0.1>0.0001$ \\
\hline 90 & 11.0016 & 8.3728 & -31.1796 & 10.6621 & 0.0938 & $0.1>0.0001$ \\
\hline
\end{tabular}

in Fig. 2 whenever $q=\left\{\frac{1}{7}, \frac{1}{2}, \frac{8}{9}\right\}$. Also, ${ }_{1} \mathbb{G}_{\mathrm{q}}(\mathfrak{t}, \mathfrak{s})$ is a continuous function for $(\mathfrak{t}, \mathfrak{s}) \in[1, \infty) \times$ $[1, \infty)$ and

$$
\left|\mathfrak{w}_{*}(\mathfrak{t}, \wp)\right| \leq \psi(\mathfrak{t}) \mathfrak{p}(|\wp|)
$$

on $J \times \mathbb{R}$ with $\mathfrak{p} \in C(J, \mathbb{R})$ nondecreasing and

$$
\int_{0}^{\infty} \psi(\xi) \mathrm{d}_{\mathrm{q}} \xi<+\infty
$$

Furthermore,

$$
\mathfrak{p}(\mu) \int_{0}^{\infty} \psi(\xi) \mathrm{d}_{\mathrm{q}} \xi=\left\{\begin{array}{ll}
0.0044, & \mathrm{q}=\frac{1}{7} \\
0.0077, & \mathrm{q}=\frac{1}{2}, \\
0.0001, & \mathrm{q}=\frac{8}{9} .
\end{array}\right\}<\mu=0.1
$$

Thus, from Theorem 3.3 we conclude that problem (24) has at least one positive solution $\wp(\mathfrak{t})$, for $\mathfrak{t} \in J$,

$$
0 \leq \frac{\wp(\mathfrak{t})}{1+\mathfrak{t}^{\frac{12}{5}}} \leq \mu .
$$

\section{Conclusion}

A fractional boundary value problem system has been proposed and studied in terms of its unbounded solutions' existence for a class of nonlinear fractional q-difference equations via the Riemann-Liouville fractional q-derivative on an infinite interval. The system's solution has been formulated using Green's function. In addition, a compactness criterion 


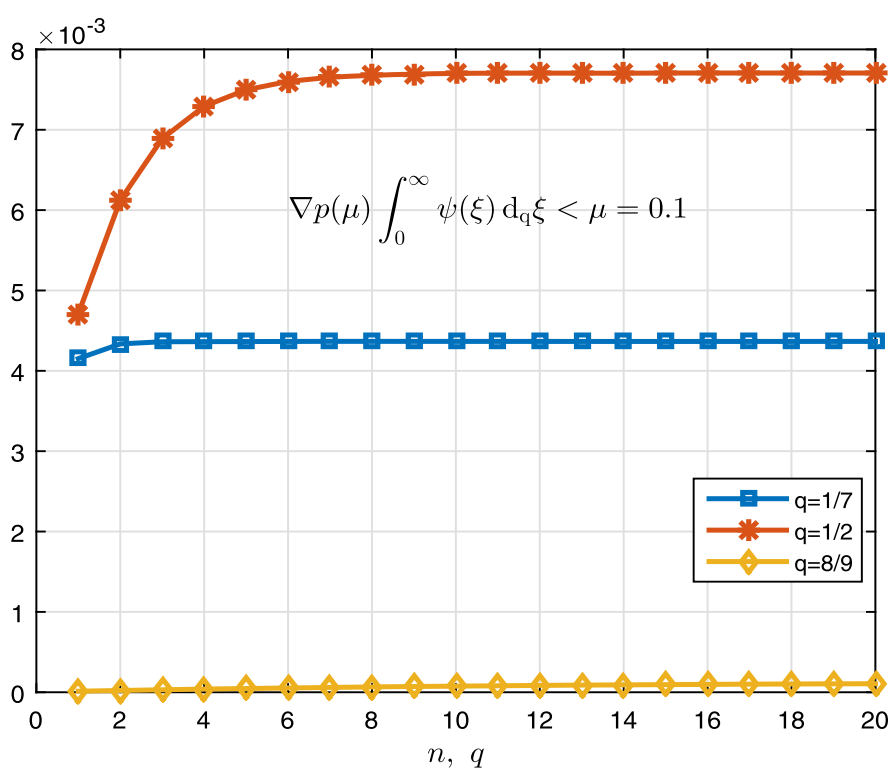

Figure 2 Graphical representation of $\nabla \mathfrak{p}(\mu) \int_{0}^{\infty} \psi(\xi) \mathrm{d}_{\mathrm{q}} \xi$ in Example 4.2

has been obtained in special space. Fixed-point theorems have been utilized to obtain all our results in this work. Illustrative examples have been provided at the end of our study.

\section{Supplementary information}

Supplementary information accompanies this paper at https://doi.org/10.1186/s13660-022-02764-6.

Additional file 1. Supplementary information (PDF 88 kB)

Acknowledgements

The fifth author was supported by Bu-Ali Sina University.

Funding

Not applicable.

Availability of data and materials

Data sharing is not applicable to this article as no datasets were generated or analyzed during the current study.

\section{Declarations}

Ethics approval and consent to participate

Not applicable.

\section{Consent for publication}

Not applicable.

\section{Competing interests}

The authors declare that they have no competing interests.

\section{Authors' contributions}

AB: Actualization, methodology, formal analysis, validation, investigation, and initial draft. MB: Actualization, validation, methodology, formal analysis, investigation, and initial draft. MKAK: Actualization, methodology, formal analysis, validation, investigation, initial draft, and supervision of the original draft and editing. FM: Actualization, validation, methodology, formal analysis, investigation, and initial draft. MES: Actualization, methodology, formal analysis, validation, investigation, software, simulation, initial draft and was a major contributor in writing the manuscript. MK: Actualization, validation, methodology, formal analysis, investigation, and initial draft. All authors read and approved the final manuscript. 


\section{Author details}

${ }^{1}$ Laboratory of Mathematics And Applied Sciences, University of Ghardaia, Ghardaia, 47000, Algeria. ${ }^{2}$ Faculty of Sciences, Saad Dahlab University, Blida, Algeria. ${ }^{3}$ Institute of Mathematical Sciences, Faculty of Science, University of Malaya, Kuala Lumpur, 50603, Malaysia. ${ }^{4}$ Department of Applied Mathematics and Statistics, Technological University of Cartagena, Cartagena, Spain. ${ }^{5}$ Department of Mathematics, Bu-Ali Sina University, Hamedan, Iran. ${ }^{6}$ Department of Computer Engineering, Kastamonu University, Kastamonu, Turkey.

\section{Publisher's Note}

Springer Nature remains neutral with regard to jurisdictional claims in published maps and institutional affiliations.

Received: 17 November 2021 Accepted: 7 February 2022 Published online: 03 March 2022

\section{References}

1. Jackson, F:: On q-definite integrals. Q. J. Pure Appl. Math. 41, 193-203 (1910)

2. Zhao, X.K., Zhao, D., Ge, W.: Solvability for fractional multi-point boundary value problems on half-line. Ann. Differ. Equ. 27(3), 395-400 (2011)

3. Kilbas, A.A., Srivastava, H.M., Trujillo, J.J.: Theory and Applications of Fractional Differential Equations. Elsevier, Amsterdam (2006)

4. Rajkovic, P.M., Marinkovic, S.D., Stankovic, M.S.: Fractional integrals and derivatives in q-calculus. Appl. Anal. Discrete Math. 1, 311-323 (2007)

5. Alzabut, J., Mohammadaliee, B., Samei, M.E.: Solutions of two fractional q-integro-differential equations under sum and integral boundary value conditions on a time scale. Adv. Differ. Equ. 2020, Article ID 304 (2020)

6. Rezapour, S., Samei, M.E.: On the existence of solutions for a multi-singular point-wise defined fractional q-integro-differential equation. Bound. Value Probl. 2020, Article ID 38 (2020)

7. Su, X., Zhang, S.: Unbounded solutions to a boundary value problem of fractional order on the half-line. Comput. Math. Appl. 61, 1079-1087 (2011)

8. Agarwal, R.P., Ahmad, B., Alsaedi, A., Al-Hutami, H.: Existence theory for q-antiperiodic boundary value problems of sequential q-fractional integro-differential equations. Abstr. Appl. Anal. 2014, Article ID 207547 (2014)

9. Agarwal, R.P.: Certain fractional q-integrals and q-derivatives. Proc. Camb. Philol. Soc. 66, 365-370 (1969)

10. Al-Salam, W.A.: Some fractional q-integrals and q-derivatives. Proc. Edinb. Math. Soc. 15, 135-140 (1966)

11. Annaby, M.H., Mansour, Z.S.: q-Fractional Calculus and Equations. Lecture Notes in Mathematics, vol. 2056. Springer, Berlin (2012)

12. Ferreira, R.A.C.: Nontrivial solutions for fractional q-difference boundary value problems. Electron. J. Qual. Theory Differ. Equ. 70, 1 (2010)

13. Etemad, S., Ntouyas, S.K., Ahmad, B.: Existence theory for a fractional q-integro-difference equation with q-integral boundary conditions of different orders. Mathematics 7, 659 (2019)

14. Wang, G., Ahmad, B., Zhang, L.: A coupled system of nonlinear fractional differential equations with multipoint fractional boundary conditions on an unbounded domain, hindawi publishing corporation. Abstr. Appl. Anal. 2012 Article ID 248709 (2012)

15. Zhang, L., Ahmad, B., Wang, G., Agarwal, R.P., Al-Yami, M., Shammakh, W.: Nonlocal integrodifferential boundary value problem for nonlinear fractional differential equations on an unbounded domain. Abstr. Appl. Anal. 2013, Article ID 813903 (2013)

16. Mason, T.E.: On properties of the solution of linear q-difference equations with entire function coefficients. Am. J. Math. 37, 439-444 (1915)

17. Trjitzinsky, W.J.: Analytic theory of linear q-difference equations. Acta Math. 61, 1-38 (1933)

18. Alizadeh, S., Baleanu, D., Rezapour, S.: Analyzing transient response of the parallel rcl circuit by using the Caputo-Fabrizio fractional derivative. Adv. Differ. Equ. 2020, Article ID 55 (2020)

19. Samei, M.E., Rezapour, S.: On a system of fractional $q$-differential inclusions via sum of two multi-term functions on a time scale. Bound. Value Probl. 2020, Article ID 135 (2020)

20. Balanu, D., Mohammadi, H., Rezapour, S.: Analysis of the model of hiv-1 infection of $c d 4^{+} t$-cell with a new approach of fractional derivative. Adv. Differ. Equ. 2020, Article ID 71 (2020)

21. Ahmad, B., Ntouyas, S., Purnaras, I.K.: Existence results for nonlocal boundary value problems of nonlinear fractional q-difference equations. Adv. Differ. Equ. 2012, Article ID 140 (2012)

22. Ahmad, B., Nieto, J.J., Alsaedi, A., Al-Hutami, H.: Boundary value problems of nonlinear fractional q-difference (integral) equations with two fractional orders and four-point nonlocal integral boundary conditions. Filomat 28(8), 1719-1736 (2014)

23. Almeida, R., Martínez, N.: Existence results for fractional q-difference equations of order $\alpha \in] 2,3[$ with three-point boundary conditions. Commun. Nonlinear Sci. Numer. Simul. 19, 1675-1685 (2014)

24. Ma, K., Li, X., Sun, S.: Boundary value problems of fractional q-difference equations on the half-line. Bound. Value Probl. 2019, Article ID 46 (2019)

25. Zhou, W.X., Liu, H.Z.: Existence solutions for boundary value problem of nonlinear fractional q-difference equations. Adv. Differ. Equ. 2013, Article ID 113 (2013)

26. Etemad, S., Rezapour, S., Samei, M.E.: $\alpha-\psi$-contractions and solutions of a q-fractional differential inclusion with three-point boundary value conditions via computational results. Adv. Differ. Equ. 2020, Article ID 218 (2020)

27. Baleanu, D., Etemad, S., Pourrazi, S., Rezapour, S.: On the new fractional hybrid boundary value problems with three-point integral hybrid conditions. Adv. Differ. Equ. 2019, Article ID 473 (2019)

28. Baleanu, D., Hedayati, V., Rezapour, S., Mohamed Al Qurashi, M.: On two fractional differential inclusions. SpringerPlus 5(1), $882(2016)$

29. Baleanu, D., Etemad, S., Rezapour, S.: On a fractional hybrid integro-differential equation with mixed hybrid integral boundary value conditions by using three operators. Alex. Eng. J. 59(5), 3019-3027 (2020) 
30. Arara, A., Benchohra, M., Hamidia, N., Nieto, J.J.: Fractional order differential equations on an unbounded domain. Nonlinear Anal. 72, 580-586 (2010)

31. Su, X.: Solutions to boundary value problem of fractional order on unbounded domains in a Banach space. Nonlinear Anal. 74, 2844-2852 (2011)

32. Zhao, X.K., Ge, W.G.: Unbounded solutions for a fractional boundary value problem on the infinite interval. Acta Appl. Math. 109, 495-505 (2010)

33. Ntouyas, S.K., Samei, M.E.: Existence and uniqueness of solutions for multi-term fractional q-integro-differential equations via quantum calculus. Adv. Differ. Equ. 2019, Article ID 475 (2019)

34. Samei, M.E., Hedayati, V., Rezapour, S.: Existence results for a fraction hybrid differential inclusion with Caputo-Hadamard type fractional derivative. Adv. Differ. Equ. 2019, Article ID 163 (2019). https://doi.org/10.1186/s13662-019-2090-8

35. Abdeljawad, T., Samei, M.E.: Applying quantum calculus for the existence of solution of $q$-integro-differential equations with three criteria. Discrete Contin. Dyn. Syst., Ser. S 14(10), 3351-3386 (2021)

36. Etemad, S.T.M.T., Etemad, S., Rezapour, S.: On a coupled Caputo conformable system of pantograph problems. Turk. J. Math. 45(1), 496-519 (2020)

37. Mohammadi, H., Kumar, S., Rezapour, S., Etemad, S.: A theoretical study of the Caputo-Fabrizio fractional modeling for hearing loss due to Mumps virus with optimal control. Chaos Solitons Fractals 144, Article ID 110668 (2021)

38. Matar, M.M., Abbas, M.I., Alzabut, J., Kaabar, M.K.A., Etemad, S., Rezapour, S.: Investigation of the p-Laplacian nonperiodic nonlinear boundary value problem via generalized Caputo fractional derivatives. Adv. Differ. Equ. 2021, Article ID 68 (2021)

39. Kac, V., Cheung, P.: Quantum Calculus. Springer, New York (2002)

40. Rajkovic, P.M., Marinkovic, S.D., Stankovic, M.S.: On q-analogues of Caputo derivative and Mittag-Leffler function. Fract. Calc. Appl. Anal. 10, 359-373 (2007)

\section{Submit your manuscript to a SpringerOpen ${ }^{\circ}$ journal and benefit from:}

- Convenient online submission

- Rigorous peer review

- Open access: articles freely available online

- High visibility within the field

- Retaining the copyright to your article

Submit your next manuscript at $\boldsymbol{~ s p r i n g e r o p e n . c o m ~}$ 